\title{
SPARSE PHASE RETRIEVAL VIA PHASELIFTOFF
}

\author{
YU XIA AND ZHIQIANG XU
}

\begin{abstract}
The aim of sparse phase retrieval is to recover a $k$-sparse signal $\mathbf{x}_{0} \in \mathbb{C}^{d}$ from quadratic measurements $\left|\left\langle\mathbf{a}_{i}, \mathbf{x}_{0}\right\rangle\right|^{2}$ where $\mathbf{a}_{i} \in \mathbb{C}^{d}, i=1, \ldots, m$. Noting $\left|\left\langle\mathbf{a}_{i}, \mathbf{x}_{0}\right\rangle\right|^{2}=\operatorname{Tr}\left(A_{i} X_{0}\right)$ with $A_{i}=\mathbf{a}_{i} \mathbf{a}_{i}^{*} \in \mathbb{C}^{d \times d}, X_{0}=\mathbf{x}_{0} \mathbf{x}_{0}^{*} \in \mathbb{C}^{d \times d}$, one can recast sparse phase retrieval as a problem of recovering a rank-one sparse matrix from linear measurements. Yin and Xin introduced PhaseLiftOff which presents a proxy of rank-one condition via the difference of trace and Frobenius norm. By adding sparsity penalty to PhaseLiftOff, in this paper, we present a novel model to recover sparse signals from quadratic measurements. Theoretical analysis shows that the solution to our model provides the stable recovery of $\mathbf{x}_{0}$ under almost optimal sampling complexity $m=O(k \log (d / k))$. The computation of our model is carried out by the difference of convex function algorithm (DCA). Numerical experiments demonstrate that our algorithm outperforms other state-of-the-art algorithms used for solving sparse phase retrieval.
\end{abstract}

\section{INTRODUCTION}

1.1. Phase retrieval. We assume that $\mathbf{x}_{0} \in \mathbb{F}^{d}$ is a target signal, where $\mathbb{F} \in\{\mathbb{R}, \mathbb{C}\}$. The aim of phase retrieval is to recover $\mathbf{x}_{0} \in \mathbb{F}^{d}$ from $\left|\left\langle\mathbf{a}_{j}, \mathbf{x}_{0}\right\rangle\right|^{2}+w_{j}, j=1, \ldots, m$, up to a unimodular constant where $\mathbf{a}_{j} \in \mathbb{F}^{d}$ are known measurement vectors and $\mathbf{w}:=\left(w_{1}, \ldots, w_{m}\right)^{T} \in \mathbb{R}^{m}$ is a noise vector. Phase retrieval is raised in many areas, such as X-ray crystallography, astronomy, quantum tomography, optics and microscopy. For convenience, let $\mathcal{A}: \mathbb{F}^{d \times d} \rightarrow \mathbb{R}^{m}$ be a linear map which is defined as

$$
\mathcal{A}(X)=\left(\mathbf{a}_{1}^{*} X \mathbf{a}_{1}, \ldots, \mathbf{a}_{m}^{*} X \mathbf{a}_{m}\right),
$$

where $X \in \mathbb{F}^{d \times d}, \mathbf{a}_{j} \in \mathbb{F}^{d}, j=1, \ldots, m$. We abuse the notation and set

$$
\mathcal{A}(\mathbf{x}):=\mathcal{A}\left(\mathbf{x x}^{*}\right)=\left(\left|\left\langle\mathbf{a}_{1}, \mathbf{x}\right\rangle\right|^{2}, \ldots,\left|\left\langle\mathbf{a}_{m}, \mathbf{x}\right\rangle\right|^{2}\right),
$$

where $\mathbf{x} \in \mathbb{F}^{d}$. With these notations, we can formulate the aim of phase retrieval as follows: To estimate the matrix $X_{0}=\mathbf{x}_{0} \mathbf{x}_{0}^{*} \in \mathbb{C}^{d \times d}$ from $\mathcal{A}\left(\mathbf{x}_{0}\right)+\mathbf{w} \in \mathbb{R}^{m}$.

For the noiseless case, to guarantee the solution $\mathcal{A}(\mathbf{x})=\mathcal{A}\left(\mathbf{x}_{0}\right)$ is unique for all $\mathbf{x}_{0} \in \mathbb{C}^{d}$, it is shown in [20] that the measurement number $m \geq 4 d-2-2 \alpha_{d}$ is necessary where $\alpha_{d}$ denotes the number of 1's in the binary of expansion of $d-1$. The authors in $[8$ proved that $m \geq 4 d-4$ generic measurement vectors $\mathbf{a}_{j} \in \mathbb{C}^{d}, j=1, \ldots, m$, are enough to guarantee the uniqueness of the solution.

2010 Mathematics Subject Classification. 94A20, 90C26.

Key words and phrases. Signal recovery, Phase retrieval, Compressed sensing, Restricted isometry property, Compressed phaseless sensing.

Yu Xia was supported by NSFC grant (11901143), Zhejiang Provincial Natural Science Foundation (LQ19A010008), Education Department of Zhejiang Province Science Foundation (Y201840082).

Zhiqiang Xu was supported by Beijing Natural Science Foundation (Z180002) and by NSFC grant (11688101). 
In [5, 6, 7], the phase retrieval was recasted as a semi-definite programming problem, i.e., the PhaseLift problem:

$$
\min _{X \in \mathbb{F}^{d \times d}} \operatorname{Tr}(X) \quad \text { s.t. } \quad \mathcal{A}(X)=\mathcal{A}\left(X_{0}\right), X \succeq 0 .
$$

In [6], it is shown that the solution to (1.2) is $X_{0}$ with high probability provided $\mathbf{a}_{j}$ is Gaussian random vector and $m=O(d \log d)$, which was reduced to $m=O(d)$ in [5]. For the aim of computation, the regularized trace-norm minimization is suggested in [6, 7]:

$$
\min _{X \succeq 0, X \in \mathbb{F}^{d \times d}} \frac{1}{2}\|\mathcal{A}(X)-\mathbf{b}\|_{2}^{2}+\lambda \operatorname{Tr}(X) .
$$

Noting that $\operatorname{Tr}(X)-\|X\|_{F} \geq 0$ and the equality holds iff $\operatorname{rank}(X)=1$, Yin and Xin suggested the following regularization problem [23], which is called as PhaseLiftOff:

$$
\min _{X \succeq 0, X \in \mathbb{F}^{d \times d}} \frac{1}{2}\|\mathcal{A}(X)-\mathbf{b}\|_{2}^{2}+\lambda\left(\operatorname{Tr}(X)-\|X\|_{F}\right) .
$$

The numerical experiments in [23] showed that PhaseLiftOff outperforms PhaseLift.

1.2. Sparse phase retrieval. In many areas, one also requires $\left\|\mathbf{x}_{0}\right\|_{0} \leq k$, i.e., the number of nonzero entries of $\mathbf{x}_{0}$ less than or equal to $k$ [13, 19, 12. The aim of sparse phase retrieval is to recover the $k$-sparse signal $\mathbf{x}_{0}$ from $\left|\left\langle\mathbf{a}_{j}, \mathbf{x}_{0}\right\rangle\right|^{2}=b_{j}, j=1, \ldots, m$.

For convenience, we set $\Sigma_{k}^{\mathbb{F}}:=\left\{\mathbf{x} \in \mathbb{F}^{d}:\|\mathbf{x}\|_{0} \leq k\right\}$. It was shown in [19] that, for $\mathbb{F}=\mathbb{C}$ and $\mathbf{x}_{0} \in \Sigma_{k}^{\mathbb{F}}$, if $m \geq 4 k-2$ (resp. $m \geq 2 k$ for $\mathbb{F}=\mathbb{R}$ ) and $\mathbf{a}_{1}, \ldots, \mathbf{a}_{m}$ are generic vectors in $\mathbb{C}^{d}$ (resp. $\mathbb{R}^{d}$ ) then the solution to $\mathcal{A}(\mathbf{x})=\mathcal{A}\left(\mathbf{x}_{0}\right)$ with $\mathbf{x} \in \Sigma_{k}^{\mathbb{F}}$ is unique up to a unimodular constant.

The $\ell_{1}$-minimization is a commonly used method for recovering sparse signals. Naturally, one is also interested in employing $\ell_{1}$-minimization for solving sparse phase retrieval. For $\mathbb{F}=\mathbb{R}$, the following model was considered in [17]:

$$
\min _{\mathbf{x} \in \mathbb{R}^{d \times d}}\|\mathbf{x}\|_{1} \quad \text { s.t. } \quad \mathcal{A}(\mathbf{x})=\mathcal{A}\left(\mathbf{x}_{0}\right) .
$$

Particularly, it is proved that the solution to (1.5) is $\pm \mathbf{x}_{0}$ with high probability if $m \gtrsim k \log d$ and $\mathbf{a}_{j}, j=1, \ldots, m$, are independent Gaussian random vectors. In [22], the authors extended this result to the case where $\mathbb{F}=\mathbb{C}$.

In [10], the following convex model was considered

$$
\min _{X \in \mathbb{R}^{d \times d}}\|X\|_{1}+\lambda \operatorname{Tr}(X), \quad \text { s.t. } \quad \mathcal{A}(X)=\mathcal{A}\left(\mathbf{x}_{0}\right), X \succeq 0 .
$$

The objective function in (1.6) is the summation of the trace and the $\ell_{1}$ norm, which is also a convex model. To guarantee the solution to (1.6) is $\mathbf{x}_{0} \mathbf{x}_{0}^{*}$, one has to require the number of measurements $m \gtrsim k^{2} \log d$, which is quadratic about the sparse level $k$ [10].

Beyond the convex model, one also develops many nonconvex algorithms for solving sparse phase retrieval, such as Sparse Truncated Amplitude flow (SPARTA) [18, Thresholded Wirtinger Flow (ThWF) 4, Sparse Wirtinger Flow (SWF) [25, Sparse Phase Retrieval via Smoothing Function (SPRSF) [1]. These algorithms include two stages: (i) Recover the support of the underlying sparse signal under some analytical rule, and construct an initialization near the ground truth signal $\mathbf{x}_{0}$; (ii) Refine the initialization by gradient-type iterations and extra truncation procedure by hard thresholding. However, to guarantee the algorithms converge to the true signal, the algorithms mentioned above require the sample complexity is $m=O\left(k^{2} \log d\right)$. 
1.3. Our contribution. A natural model for sparse phase retrieval is to use $\ell_{1}$-regularization methods, i.e.,

$$
\min _{X \in \mathbb{C}^{d \times d}} \mu\|X\|_{1}+\frac{1}{2}\|\mathcal{A}(X)-\mathbf{b}\|_{2}^{2}, \quad \text { s.t. } \quad X \succeq 0, \operatorname{rank}(X)=1,
$$

where $\|X\|_{1}=\sum_{l=1}^{d} \sum_{j=1}^{d}\left|x_{j, l}\right|$ and $x_{j, l}$ are the entries of $X$. Motivated by the notable PhaseLiftOff [23], we reformulate (1.7) as the following regularization problem:

$$
\min _{X \in \mathbb{C}^{d \times d}} \lambda\left(\operatorname{Tr}(X)-\|X\|_{F}\right)+\mu\|X\|_{1}+\frac{1}{2}\|\mathcal{A}(X)-\mathbf{b}\|_{2}^{2} \quad \text { s.t. } \quad X \succeq 0 .
$$

For convenience, we call (1.8) Sparse PhaseLiftOff model. Note that the object function in (1.8) is the difference of convex functions and hence it can be solved by the difference of convex functions algorithm (DCA).

To study the performance of (1.8), we first establish the equivalence between (1.8) and (1.7) under some mild conditions about $\lambda, \mu$ and $\|\mathbf{w}\|_{2}$ :

Lemma 1.1. Assume that $\mathbf{b}=\mathcal{A}\left(\mathbf{x}_{0} \mathbf{x}_{0}^{*}\right)+\mathbf{w}$ where $\mathbf{x}_{0} \in \mathbb{C}^{d}$, and $\mathbf{w} \in \mathbb{R}^{m}$ is the noise term. Let $X^{\#}$ be the global minimizer of (1.8). If $\frac{1}{2}\|\mathbf{b}\|_{2}^{2}>\mu\left\|\mathbf{x}_{0}\right\|_{1}^{2}+\frac{1}{2}\|\mathbf{w}\|_{2}^{2}, \mu \geq 0$ and

$$
\lambda>\frac{\mu d+\|\mathcal{A}\|\left(\sqrt{2 \mu}\left\|\mathbf{x}_{0}\right\|_{1}+\|\mathbf{w}\|_{2}\right)}{\sqrt{2}-1},
$$

then $\operatorname{rank}\left(X^{\#}\right)=1$

As said before, $\operatorname{Tr}(X)-\|X\|_{F}=0$ provided $\operatorname{rank}(X)=1$. Under the conditions of Lemma 1.1. $X^{\#}$ is also the minimizer of (1.7). Hence, we turn to study the performance of (1.7). To do that, we require $\mathcal{A}$ satisfies restricted isometry property over low-rank and sparse matrices:

Definition 1.1. 22 We say that the map $\mathcal{A}: \mathbb{H}^{d \times d} \rightarrow \mathbb{R}^{m}$ satisfies the restricted isometry property of order $(r, s)$ if there exist positive constants $c$ and $C$ such that the inequality

$$
c\|X\|_{F} \leq \frac{1}{m}\|\mathcal{A}(X)\|_{1} \leq C\|X\|_{F}
$$

holds for all $X \in \mathbb{H}^{d \times d}$ with $\operatorname{rank}(X) \leq r$ and $\|X\|_{0,2} \leq s$.

Then, we have the following theorem.

Theorem 1.2. Let $\mathbf{b}=\mathcal{A}\left(\mathbf{x}_{0} \mathbf{x}_{0}^{*}\right)+\mathbf{w}$, where $\mathbf{x}_{0} \in \mathbb{C}^{d}$, and $\mathbf{w} \in \mathbb{R}^{m}$ is the noise term. Assume that $\mathcal{A}(\cdot)$ satisfy the $R I P$ condition of order $(2,2 a k)$ with $R I P$ constant $c, C>0$, and $a \geq 1$ with

$$
c-\frac{4 \sqrt{2} C}{\sqrt{a}}-\frac{C}{a}>0 .
$$

Set $\alpha:=\frac{1+\frac{1}{a}+\frac{4 \sqrt{2}}{\sqrt{a}}}{c-\frac{C}{a}-\frac{4 \sqrt{2} C}{\sqrt{a}}}$. Assume that $\mu>0$. For any $k$-sparse signals $\mathbf{x}_{0} \in \mathbb{C}^{d}$, the solution to 1.7 $X^{\#}:=\mathbf{x}^{\#}\left(\mathbf{x}^{\#}\right)^{*}$ satisfies

$$
\left\|\mathbf{x}^{\#}\left(\mathbf{x}^{\#}\right)^{*}-\mathbf{x}_{0} \mathbf{x}_{0}^{*}\right\|_{F} \leq(2 C \alpha+2) \frac{\|\mathbf{w}\|_{2}}{\sqrt{\mu a k}}\left(\left\|\mathbf{x}_{0}\right\|_{1}+\frac{\|\mathbf{w}\|_{2}}{\sqrt{\mu}}\right)+\frac{\|\mathbf{w}\|_{2}^{2}}{2 \mu a k}+\alpha \cdot \frac{\mu a k}{2 C}\left(\frac{C\|\mathbf{w}\|_{2}}{\mu a k}+\frac{1}{\sqrt{m}}\right)^{2} .
$$

Combing Lemma 1.1. Theorem 1.2 and Theorem 2.1, we have the following corollary: 
Corollary 1.3. Assume that $\mathbf{a}_{j}, j=1, \ldots, m$ are independently complex Gaussian random vectors, i.e., $\mathbf{a}_{j} \sim \mathcal{N}\left(0, \frac{1}{2} \mathbf{I}_{d}\right)+\mathcal{N}\left(0, \frac{1}{2} \mathbf{I}_{d}\right)$ i. Assume that $\mathbf{b}=\mathcal{A}\left(\mathbf{x}_{0} \mathbf{x}_{0}^{*}\right)+\mathbf{w}$ where $\mathbf{w} \in \mathbb{R}^{m}$ is a noise vector and $\mathbf{x}_{0} \in \mathbb{C}^{d}$ with $\left\|\mathbf{x}_{0}\right\|_{0} \leq k$. Assume that $m \gtrsim k \log (e d / k)$. Let $X^{\#}$ be the global minimizer of model (1.8). The following holds with probability at least $1-\exp (-c m)$ : If $\frac{1}{2}\|\mathbf{b}\|_{2}^{2}>\mu\left\|\mathbf{x}_{0}\right\|_{1}^{2}+\frac{1}{2}\|\mathbf{w}\|_{2}^{2}$, $\mu>0$ and

$$
\lambda>\frac{\mu d+\|\mathcal{A}\|\left(\sqrt{2 \mu}\left\|\mathbf{x}_{0}\right\|_{1}+\|\mathbf{w}\|_{2}\right)}{\sqrt{2}-1},
$$

then $\operatorname{rank}\left(X^{\#}\right)=1$, and $X^{\#}=\mathbf{x}^{\#}\left(\mathbf{x}^{\#}\right)^{*}$ satisfies

$$
\left\|\mathbf{x}^{\#}\left(\mathbf{x}^{\#}\right)^{*}-\mathbf{x}_{0} \mathbf{x}_{0}^{*}\right\|_{F} \lesssim \frac{\|\mathbf{w}\|_{2}}{\sqrt{\mu k}}\left(\left\|\mathbf{x}_{0}\right\|_{1}+\frac{\|\mathbf{w}\|_{2}}{\sqrt{\mu}}\right)+\frac{\|\mathbf{w}\|_{2}^{2}}{2 \mu k}+\mu k\left(\frac{\|\mathbf{w}\|_{2}}{\mu k}+\frac{1}{\sqrt{m}}\right)^{2} .
$$

Remark 1.4. According to Corollary 1.3, the parameter $\lambda$ depends on $\|\mathcal{A}\|$. We next show $\|\mathcal{A}\|=$ $O((m+d) \sqrt{d})$. We assume that the singular value decomposition of $X \in \mathbb{C}^{d \times d}$ with $\|X\|_{F}=1$ is $X=\sum_{j=1}^{d} \sigma_{j} \mathbf{u}_{j} \mathbf{v}_{j}^{*}$. Here, $\sum_{j=1}^{d} \sigma_{j}^{2}=1$. We claim that $\left\|\mathcal{A}\left(\mathbf{u}_{j} \mathbf{v}_{j}^{*}\right)\right\|_{1}=O(m+d)$ holds with probability at least $1-\exp (-m)$. Then we have

$$
\|\mathcal{A}(X)\|_{2} \leq\|\mathcal{A}(X)\|_{1} \leq \sum_{j} \sigma_{j}\left\|\mathcal{A}\left(\mathbf{u}_{j} \mathbf{v}_{j}^{*}\right)\right\|_{1}=O((m+d) \sqrt{d}) .
$$

Note that

$$
\left\|\mathcal{A}\left(\mathbf{u}_{j} \mathbf{v}_{j}^{*}\right)\right\|_{1}=\sum_{i}\left|\left\langle\mathbf{a}_{i}, \mathbf{u}_{j}\right\rangle\left\langle\mathbf{a}_{i}, \mathbf{v}_{j}\right\rangle\right| \leq \sqrt{\sum_{j}\left|\left\langle\mathbf{a}_{i}, \mathbf{u}_{j}\right\rangle\right|^{2}} \sqrt{\sum_{j}\left|\left\langle\mathbf{a}_{i}, \mathbf{v}_{j}\right\rangle\right|^{2}} \leq(\sqrt{m}+\sqrt{d}+t)^{2}
$$

holds with probability larger than $1-\exp \left(-t^{2} / 2\right)$. Here, the last inequality follows from the singular values of Gaussian random matrices [16, Corrollary 5.35]. By taking $t=\sqrt{m}+\sqrt{d}$, we have $\left\|\mathcal{A}\left(\mathbf{u}_{j} \mathbf{v}_{j}^{*}\right)\right\|_{1}=O(m+d)$.

Remark 1.5. If we take $\|\mathbf{w}\|_{2}=0$ in Corollary 1.3 then the following holds with high probability:

$$
\left\|\mathbf{x}^{\#}\left(\mathbf{x}^{\#}\right)^{*}-\mathbf{x}_{0} \mathbf{x}_{0}^{*}\right\|_{2} \lesssim \frac{\mu k}{m}
$$

provided

$$
\lambda>\frac{\mu d+\|\mathcal{A}\|\left(\sqrt{2 \mu}\left\|\mathbf{x}_{0}\right\|_{1}\right)}{\sqrt{2}-1}
$$

1.4. Notations. We use $\mathbb{H}^{d \times d}$ to denote the set of all $d \times d$ Hermitian matrices. For any $X, Y \in$ $\mathbb{H}^{d \times d}$, set $\langle X, Y\rangle:=\operatorname{Tr}\left(X^{*} Y\right)$. For $x \in \mathbb{C}$, we use $\mathscr{R}(x)$ and $\mathscr{I}(x)$ to denote the real and complex parts of $x$, respectively. For $X \in \mathbb{C}^{d \times d}$, we use $X_{i,:}$ and $X_{:, j}$ to denote the $i$-th row and $j$-th column of $X$, respectively. For $S, T \subset\{1, \ldots, d\}$, we use $X_{S, T}$ to denote a submatrix of $X$ with the rows indexed in $S$ and columns indexed in $T$. We also set $\|X\|_{1}:=\sum_{i, j} \sqrt{\mathscr{R}\left(X_{i, j}\right)^{2}+\mathscr{I}\left(X_{i, j}\right)^{2}}$, $\|X\|_{F}:=\sqrt{\sum_{i, j}\left(\mathscr{R}\left(X_{i, j}\right)^{2}+\mathscr{I}\left(X_{i, j}\right)^{2}\right)}$, and $\|X\|_{1,2}:=\sum_{j}\left\|X_{:, j}\right\|_{2}$. We use $\|X\|_{0,2}$ to denote the number of non-zero columns in $X$ and use $\operatorname{vec}(X) \in \mathbb{C}^{d^{2}}$ to denote the vectorization of $X \in \mathbb{C}^{d \times d}$.

1.5. Organization. The paper is organized as follows. After introducing some useful lemmas in Section 2, we present the proof of Theorem 1.2 in Section 3. The proof of Lemma 1.1 is presented in Section 4. In Section 5, we make a lot of numerical experiments, which show our method has better performance over the other known algorithms for sparse phase retrieval. 


\section{Preliminaries and Lemmas}

The following theorem shows that complex Gaussian random quadratic map $\mathcal{A}$ satisfies RIP of order $(2, s)$ with high probability provided $m \gtrsim s \log (d / s)$.

Theorem 2.1. 22] Assume that the linear measurement $\mathcal{A}(\cdot)$ is defined as

$$
\mathcal{A}(X)=\left(\mathbf{a}_{1}^{*} X \mathbf{a}_{1}, \ldots, \mathbf{a}_{m}^{*} X \mathbf{a}_{m}\right),
$$

where $\mathbf{a}_{j}$ are independently complex Gaussian random vectors, i.e., $\mathbf{a}_{j} \sim \mathcal{N}\left(0, \frac{1}{2} \mathbf{I}_{n \times n}\right)+\mathcal{N}\left(0, \frac{1}{2} \mathbf{I}_{n \times n}\right) i$. If

$$
m \gtrsim s \log (d / s),
$$

under the probability at least $1-2 \exp \left(-c_{0} m\right)$, the linear map $\mathcal{A}$ satisfies the restricted isometry property of order $(2, s)$, i.e.

$$
0.12\|X\|_{F} \leq \frac{1}{m}\|\mathcal{A}(X)\|_{1} \leq 2.45\|X\|_{F}
$$

for all $X \in \mathbb{H}^{n \times n}$ with $\operatorname{rank}(X) \leq 2$ and $\|X\|_{0,2} \leq s$ (also $\left\|X^{*}\right\|_{0,2} \leq s$ ).

We also need the following lemma.

Lemma 2.2. 22] If $\mathbf{x}, \mathbf{y} \in \mathbb{C}^{d}$, and $\langle\mathbf{x}, \mathbf{y}\rangle \geq 0$. Then

$$
\left\|\mathbf{x} \mathbf{x}^{*}-\mathbf{y y}^{*}\right\|_{F}^{2} \geq \frac{1}{2}\|\mathbf{x}\|_{2}^{2}\|\mathbf{x}-\mathbf{y}\|_{2}^{2}
$$

The following lemma follows from the proof of Theorem 3.1 in [23. We include a proof here for completeness.

Lemma 2.3. Suppose that $\langle X, Y\rangle=0$, where $X, Y \succeq 0$. If $\operatorname{rank}(X)=r \geq 1$, then

$$
\left\|\lambda\left(\mathbf{I}-\frac{X}{\|X\|_{F}}\right)-Y\right\|_{F} \geq \lambda(\sqrt{r}-1) .
$$

Here $\lambda$ is a non-negative constant.

Proof. First of all, we have

$$
\left\|\lambda\left(\mathbf{I}-\frac{X}{\|X\|_{F}}\right)-Y\right\|_{F} \geq\|\lambda \mathbf{I}-Y\|_{F}-\lambda\left\|\frac{X}{\|X\|_{F}}\right\|_{F}=\|\lambda \mathbf{I}-Y\|_{F}-\lambda,
$$

Then we estimate the lower bound of $\|\lambda \mathbf{I}-Y\|_{F}$. Suppose that the singular value decomposition of $X$ is in the form of

$$
X=\sum_{i=1}^{r} \sigma_{i} \mathbf{u}_{i} \mathbf{u}_{i}^{*}
$$

where $U_{1}=\left(\mathbf{u}_{1}, \ldots, \mathbf{u}_{r}\right) \in \mathbb{C}^{d \times r}$, and $\sigma_{i}>0$, for $i=1, \ldots, r$. Construct $U_{2} \in \mathbb{C}^{d \times(d-r)}$, which satisfies $\mathbf{I}=U_{1} U_{1}^{*}+U_{2} U_{2}^{*}$ and $U_{1}^{*} U_{2}=0$. Then we have

$$
\begin{aligned}
\|\lambda \mathbf{I}-Y\|_{F}^{2} & =\left\|\lambda\left(U_{1} U_{1}^{*}+U_{2} U_{2}^{*}\right)-Y\right\|_{F}^{2}=\left\|\lambda U_{1} U_{1}^{*}+\left(\lambda U_{2} U_{2}^{*}-Y\right)\right\|_{F}^{2} \\
& =\left\|\lambda U_{1} U_{1}^{*}\right\|_{F}^{2}+\left\|\lambda U_{2} U_{2}^{*}-Y\right\|_{F}^{2}+2 \lambda\left\langle U_{1} U_{1}^{*}, \lambda U_{2} U_{2}^{*}-Y\right\rangle \\
& =\left\|\lambda U_{1} U_{1}^{*}\right\|_{F}^{2}+\left\|\lambda U_{2} U_{2}^{*}-Y\right\|_{F}^{2}-2 \lambda\left\langle U_{1} U_{1}^{*}, Y\right\rangle .
\end{aligned}
$$

The last line follows from $U_{1}^{*} U_{2}=0$. Since $\sigma_{i}>0$ and $Y \succeq 0$, the condition $\langle X, Y\rangle=0$ implies that

$$
0=\langle X, Y\rangle=\sum_{i=1}^{r} \sigma_{i}\left\langle\mathbf{u}_{i} \mathbf{u}_{i}^{*}, Y\right\rangle=\sum_{i=1}^{r} \sigma_{i} \operatorname{Tr}\left(\mathbf{u}_{i}^{*} Y \mathbf{u}_{i}\right),
$$


which leads to $\left\langle\mathbf{u}_{i} \mathbf{u}_{i}^{*}, Y\right\rangle=0, i=1, \ldots, r$. Therefore, it obtain that

$$
\left\langle U_{1} U_{1}^{*}, Y\right\rangle=\sum_{i=1}^{r}\left\langle\mathbf{u}_{i} \mathbf{u}_{i}^{*}, Y\right\rangle=0
$$

and (2.3) becomes

$$
\|\lambda \mathbf{I}-Y\|_{F}^{2}=\left\|\lambda U_{1} U_{1}^{*}\right\|_{F}^{2}+\left\|\lambda U_{2} U_{2}^{*}-Y\right\|_{F}^{2} \geq\left\|\lambda U_{1} U_{1}^{*}\right\|_{F}^{2}=\lambda^{2} r .
$$

Combining (2.2) and (2.5), we have

$$
\left\|\lambda\left(\mathbf{I}-\frac{X}{\|X\|_{F}}\right)-Y\right\|_{F} \geq\|\lambda \mathbf{I}-Y\|_{F}-\lambda \geq \lambda(\sqrt{r}-1) .
$$

\section{Proof of Theorem 1.2}

The aim of this section is to present the proof of Theorem 1.2 .

Proof of Theorem 1.2. Set

$$
\mathbf{x}^{\#}:=\underset{\mathbf{x} \in \mathbb{C}^{d}}{\operatorname{argmin}} \mu\|\mathbf{x}\|_{1}^{2}+\frac{1}{2} \sum_{i=1}^{m}\left(\left|\left\langle\mathbf{a}_{i}, \mathbf{x}\right\rangle\right|^{2}-b_{i}\right)^{2} .
$$

Noting $\exp (i \theta) \mathbf{x}^{\#}$ is also a solution to 3.1 for any $\theta \in \mathbb{R}$, without loss of generality, we can assume that

$$
\left\langle\mathbf{x}^{\#}, \mathbf{x}_{0}\right\rangle \in \mathbb{R} \quad \text { and } \quad\left\langle\mathbf{x}^{\#}, \mathbf{x}_{0}\right\rangle \geq 0 .
$$

Then a simple observation is that $X^{\#}$ is the solution to (1.7) if and only if $X^{\#}=\mathbf{x}^{\#}\left(\mathbf{x}^{\#}\right)^{*}$.

Set $X_{0}:=\mathbf{x}_{0} \mathbf{x}_{0}^{*}$ and

$$
H:=X^{\#}-X_{0}=\mathbf{x}^{\#}\left(\mathbf{x}^{\#}\right)^{*}-\mathbf{x}_{0} \mathbf{x}_{0}^{*} .
$$

To prove the conlusion, it is enough to consider the upper bound of $\|H\|_{F}$. Set $T_{0}:=\operatorname{supp}\left(\mathbf{x}_{0}\right)$. Set $T_{1}$ as the index set which contains the indices of the $a k$ largest elements of $\mathbf{x}_{T_{0}^{c}}^{\#}$ in magnitude, and $T_{2}$ contains the indices of the next $a k$ largest elements, and so on. For simplicity, we set $T_{01}:=T_{0} \cup T_{1}$ and $\bar{H}:=H_{T_{01}, T_{01}}$. Note that

$$
\|H\|_{F} \leq\|\bar{H}\|_{F}+\sum_{i \geq 2, j \geq 2}\left\|H_{T_{i}, T_{j}}\right\|_{F}+2 \sum_{j \geq 2, i=0,1}\left\|H_{T_{i}, T_{j}}\right\|_{F} .
$$

So, it is enough to present upper bounds for

$$
\|\bar{H}\|_{F}, \sum_{i \geq 2, j \geq 2}\left\|H_{T_{i}, T_{j}}\right\|_{F}, \text { and } \sum_{j \geq 2, i=0,1}\left\|H_{T_{i}, T_{j}}\right\|_{F} .
$$

We first consider $\sum_{i \geq 2, j \geq 2}\left\|H_{T_{i}, T_{j}}\right\|_{F}$. According to

$$
\mu\left\|X^{\#}\right\|_{1}+\frac{1}{2}\left\|\mathcal{A}\left(X^{\#}\right)-\mathbf{b}\right\|_{2}^{2} \leq \mu\left\|X_{0}\right\|_{1}+\frac{1}{2}\left\|\mathcal{A}\left(X_{0}\right)-\mathbf{b}\right\|_{2}^{2},
$$

we can obtain that

$$
\mu\left\|H-H_{T_{0}, T_{0}}\right\|_{1} \leq \mu\left\|H_{T_{0}, T_{0}}\right\|_{1}+\|\mathbf{w}\|_{2}\|\mathcal{A}(H)\|_{2}-\frac{1}{2}\|\mathcal{A}(H)\|_{2}^{2} .
$$

Here, we use

$$
\left\|X_{0}\right\|_{1}=\left\|H_{T_{0}, T_{0}}-X_{T_{0}, T_{0}}^{\#}\right\|_{1} \leq\left\|H_{T_{0}, T_{0}}\right\|_{1}+\left\|X_{T_{0}, T_{0}}^{\#}\right\|_{1},
$$


and

$$
\left\|X^{\#}\right\|_{1}-\left\|X_{T_{0}, T_{0}}^{\#}\right\|_{1}=\left\|X^{\#}-X_{T_{0}, T_{0}}^{\#}\right\|_{1}=\left\|H-H_{T_{0}, T_{0}}\right\|_{1} .
$$

Therefore, we have

$$
\begin{aligned}
\sum_{i \geq 2, j \geq 2}\left\|H_{T_{i}, T_{j}}\right\|_{F} & =\sum_{i \geq 2, j \geq 2}\left\|\mathbf{x}_{T_{i}}^{\#}\right\|_{2} \cdot\left\|\mathbf{x}_{T_{j}}^{\#}\right\|_{2}=\left(\sum_{i \geq 2}\left\|\mathbf{x}_{T_{i}}^{\#}\right\|_{2}\right)^{2} \\
& \leq \frac{1}{a k}\left\|\mathbf{x}_{T_{0}^{c}}^{\#}\right\|_{1}^{2}=\frac{1}{a k}\left\|H_{T_{0}^{c}, T_{0}^{c}}\right\|_{1} \leq \frac{1}{a k}\left\|H-H_{T_{0}, T_{0}}\right\|_{1} \\
& \leq \frac{1}{a k}\left\|H_{T_{0}, T_{0}}\right\|_{1}+\frac{\|\mathbf{w}\|_{2}}{\mu a k}\|\mathcal{A}(H)\|_{2}-\frac{1}{2 \mu a k}\|\mathcal{A}(H)\|_{2}^{2} \\
& \leq \frac{1}{a}\left\|H_{T_{0}, T_{0}}\right\|_{F}+\frac{\|\mathbf{w}\|_{2}}{\mu a k}\|\mathcal{A}(H)\|_{2}-\frac{1}{2 \mu a k}\|\mathcal{A}(H)\|_{2}^{2} \\
& \leq \frac{1}{a}\|\bar{H}\|_{F}+\frac{\|\mathbf{w}\|_{2}}{\mu a k}\|\mathcal{A}(H)\|_{2}-\frac{1}{2 \mu a k}\|\mathcal{A}(H)\|_{2}^{2} .
\end{aligned}
$$

The second line based on $\left\|\mathbf{x}_{T_{j}}^{\#}\right\|_{2} \leq\left\|\mathbf{x}_{T_{j-1}}^{\#}\right\|_{1} / \sqrt{a k}$, and the third line follows from (3.2).

Second, we consider $\sum_{j \geq 2, i=0,1}\left\|H_{T_{i}, T_{j}}\right\|_{F}$. Applying that $\left\|\mathbf{x}_{T_{j}}^{\#}\right\|_{2} \leq\left\|\mathbf{x}_{T_{j-1}}^{\#}\right\|_{1} / \sqrt{a k}$, we have

$$
\begin{aligned}
\sum_{j \geq 2}\left\|H_{T_{i}, T_{j}}\right\|_{F} & =\left\|\mathbf{x}_{T_{i}}^{\#}\right\|_{2} \cdot \sum_{j \geq 2}\left\|\mathbf{x}_{T_{j}}^{\#}\right\|_{2} \leq \frac{1}{\sqrt{a k}}\left\|\mathbf{x}_{T_{0}^{c}}^{\#}\right\|_{1}\left\|\mathbf{x}_{T_{i}}^{\#}\right\|_{2} \\
& \leq \frac{1}{\sqrt{a}}\left\|\mathbf{x}_{T_{01}}^{\#}-\mathbf{x}_{0}\right\|_{2}\left\|\mathbf{x}_{T_{i}}^{\#}\right\|_{2}+\frac{\|\mathbf{w}\|_{2}}{\sqrt{2 \mu a k}}\left\|\mathbf{x}_{T_{i}}^{\#}\right\|_{2} \\
& \leq \frac{\sqrt{2}}{\sqrt{a}}\left\|\mathbf{x}_{T_{01}}^{\#}\left(\mathbf{x}_{T_{01}}^{\#}\right)^{*}-\mathbf{x}_{0} \mathbf{x}_{0}^{*}\right\|_{F}+\frac{\|\mathbf{w}\|_{2}}{\sqrt{2 \mu a k}}\left\|\mathbf{x}_{T_{i}}^{\#}\right\|_{2} \\
& =\frac{\sqrt{2}}{\sqrt{a}}\|\bar{H}\|_{F}+\frac{\|\mathbf{w}\|_{2}}{\sqrt{2 \mu a k}}\left\|\mathbf{x}_{T_{i}}^{\#}\right\|_{2},
\end{aligned}
$$

where $i \in\{0,1\}$. Here, the third line follows from Lemma 2.2 and the second line follows from

$$
\left\|\mathbf{x}_{T_{0}^{c}}^{\#}\right\|_{1} \leq \sqrt{k}\left\|\mathbf{x}_{T_{0}}^{\#}-\mathbf{x}_{0}\right\|_{2}+\frac{\|\mathbf{w}\|_{2}}{\sqrt{2 \mu}}
$$

Indeed, noting that

$$
\mu\left\|\mathbf{x}^{\#}\left(\mathbf{x}^{\#}\right)^{*}\right\|_{1}+\frac{1}{2}\left\|\mathcal{A}\left(\mathbf{x}^{\#}\left(\mathbf{x}^{\#}\right)^{*}\right)-\mathbf{b}\right\|_{2}^{2} \leq \mu\left\|\mathbf{x}_{0} \mathbf{x}_{0}^{*}\right\|_{1}+\frac{1}{2}\left\|\mathcal{A}\left(\mathbf{x}_{0}\left(\mathbf{x}_{0}\right)^{*}\right)-\mathbf{b}\right\|_{2}^{2},
$$

we obtain that

$$
\mu\left\|\mathbf{x}^{\#}\right\|_{1}^{2}+\frac{1}{2}\left\|\mathcal{A}\left(\mathbf{x}^{\#}\left(\mathbf{x}^{\#}\right)^{*}\right)-\mathbf{b}\right\|_{2}^{2} \leq \mu\left\|\mathbf{x}_{0}\right\|_{1}^{2}+\frac{1}{2}\left\|\mathcal{A}\left(\mathbf{x}_{0}\left(\mathbf{x}_{0}\right)^{*}\right)-\mathbf{b}\right\|_{2}^{2}
$$


which implies

$$
\begin{aligned}
\left\|\mathrm{x}^{\#}\right\|_{1} & \leq \sqrt{\left\|\mathbf{x}_{0}\right\|_{1}^{2}+\frac{1}{2 \mu}\left\|\mathcal{A}\left(\mathbf{x}_{0}\left(\mathbf{x}_{0}\right)^{*}\right)-\mathbf{b}\right\|_{2}^{2}-\frac{1}{2 \mu}\left\|\mathcal{A}\left(\mathrm{x}^{\#}\left(\mathbf{x}^{\#}\right)^{*}\right)-\mathbf{b}\right\|_{2}^{2}} \\
& =\sqrt{\left\|\mathbf{x}_{0}\right\|_{1}^{2}+\frac{1}{2 \mu}\left\|\mathcal{A}\left(\mathbf{x}_{0}\left(\mathbf{x}_{0}\right)^{*}\right)-\mathbf{b}\right\|_{2}^{2}-\frac{1}{2 \mu}\left\|\mathcal{A}\left(\mathbf{x}^{\#}\left(\mathbf{x}^{\#}\right)^{*}\right)-\mathcal{A}\left(\mathbf{x}_{0}\left(\mathbf{x}_{0}\right)^{*}\right)+\mathcal{A}\left(\mathbf{x}_{0}\left(\mathbf{x}_{0}\right)^{*}\right)-\mathbf{b}\right\|_{2}^{2}} \\
& =\sqrt{\left\|\mathbf{x}_{0}\right\|_{1}^{2}-\frac{1}{2 \mu}\left\|\mathcal{A}\left(\mathbf{x}_{0}\left(\mathbf{x}_{0}\right)^{*}-\mathbf{x}^{\#}\left(\mathbf{x}^{\#}\right)^{*}\right)\right\|_{2}^{2}-\frac{1}{\mu}\left\langle\mathcal{A}\left(\mathbf{x}_{0}\left(\mathbf{x}_{0}\right)^{*}-\mathbf{x}^{\#}\left(\mathbf{x}^{\#}\right)^{*}\right), \mathcal{A}\left(\mathbf{x}_{0}\left(\mathbf{x}_{0}\right)^{*}\right)-\mathbf{b}\right\rangle} \\
& \leq \sqrt{\left\|\mathbf{x}_{0}\right\|_{1}^{2}+\frac{1}{\mu}\|\mathcal{A}(H)\|_{2}\left\|\mathcal{A}\left(\mathbf{x}_{0}\left(\mathbf{x}_{0}\right)^{*}\right)-\mathbf{b}\right\|_{2}-\frac{1}{2 \mu}\|\mathcal{A}(H)\|_{2}^{2}} \\
& =\sqrt{\left\|\mathbf{x}_{0}\right\|_{1}^{2}+\frac{\|\mathbf{w}\|_{2}}{\mu}\|\mathcal{A}(H)\|_{2}-\frac{1}{2 \mu}\|\mathcal{A}(H)\|_{2}^{2}} \\
& \leq\left\|\mathbf{x}_{0}\right\|_{1}+\sqrt{\max \left\{0, \frac{\|\mathbf{w}\|_{2}}{\mu}\|\mathcal{A}(H)\|_{2}-\frac{1}{2 \mu}\|\mathcal{A}(H)\|_{2}^{2}\right\}} \\
& \leq\left\|\mathbf{x}_{0}\right\|_{1}+\frac{\|\mathbf{w}\|_{2}}{\sqrt{2 \mu}} .
\end{aligned}
$$

Therefore, we have

$$
\left\|\mathbf{x}_{T_{0}^{c}}^{\#}\right\|_{1} \leq-\left\|\mathbf{x}_{T_{0}}^{\#}\right\|_{1}+\left\|\mathbf{x}_{0}\right\|_{1}+\frac{\|\mathbf{w}\|_{2}}{\sqrt{2 \mu}} \leq\left\|\mathbf{x}_{T_{0}}^{\#}-\mathbf{x}_{0}\right\|_{1}+\frac{\|\mathbf{w}\|_{2}}{\sqrt{2 \mu}} \leq \sqrt{k}\left\|\mathbf{x}_{T_{0}}^{\#}-\mathbf{x}_{0}\right\|_{2}+\frac{\|\mathbf{w}\|_{2}}{\sqrt{2 \mu}},
$$

which implies (3.5). Combing (3.3) and (3.4), we have

$$
\begin{aligned}
& \sum_{i \geq 2, j \geq 2}\left\|H_{T_{i}, T_{j}}\right\|_{F}+2 \sum_{j \geq 2, i=0,1}\left\|H_{T_{i}, T_{j}}\right\|_{F} \\
\leq & \left(\frac{1}{a}+\frac{4 \sqrt{2}}{\sqrt{a}}\right)\|\bar{H}\|_{F}+\frac{2\|\mathbf{w}\|_{2}}{\sqrt{\mu a k}}\left\|\mathbf{x}_{T_{01}}^{\#}\right\|_{2}+\frac{\|\mathbf{w}\|_{2}}{\mu a k}\|\mathcal{A}(H)\|_{2}-\frac{1}{2 \mu a k}\|\mathcal{A}(H)\|_{2}^{2} \\
\leq & \left(\frac{1}{a}+\frac{4 \sqrt{2}}{\sqrt{a}}\right)\|\bar{H}\|_{F}+\frac{2\|\mathbf{w}\|_{2}}{\sqrt{\mu a k}}\left\|\mathbf{x}_{T_{01}}^{\#}\right\|_{2}+\frac{\|\mathbf{w}\|_{2}^{2}}{2 \mu a k} .
\end{aligned}
$$

Third, we claim that

$$
\|\bar{H}\|_{F} \leq \frac{1}{c-\frac{C}{a}-\frac{4 \sqrt{2} C}{\sqrt{a}}}\left(2 C \frac{\|\mathbf{w}\|_{2}}{\sqrt{\mu a k}}\left\|\mathbf{x}_{T_{01}}^{\#}\right\|_{2}+\frac{\mu a k}{2 C}\left(\frac{C\|\mathbf{w}\|_{2}}{\mu a k}+\frac{1}{\sqrt{m}}\right)^{2}\right) .
$$

Combining (3.7) and (3.8), we obtain that

$$
\begin{aligned}
\|H\|_{F} & \leq\|\bar{H}\|_{F}+\sum_{i \geq 2, j \geq 2}\left\|H_{T_{i}, T_{j}}\right\|_{F}+2 \sum_{j \geq 2, i=0,1}\left\|H_{T_{i}, T_{j}}\right\|_{F} \\
& \leq\left(1+\frac{1}{a}+\frac{4 \sqrt{2}}{\sqrt{a}}\right)\|\bar{H}\|_{F}+\frac{2\|\mathbf{w}\|_{2}}{\sqrt{\mu a k}}\left\|\mathbf{x}_{T_{01}}^{\#}\right\|_{2}+\frac{\|\mathbf{w}\|_{2}^{2}}{2 \mu a k} \\
& \leq(2 C \alpha+2) \frac{\|\mathbf{w}\|_{2}}{\sqrt{\mu a k}}\left\|\mathbf{x}_{T_{01}}^{\#}\right\|_{2}+\frac{\|\mathbf{w}\|_{2}^{2}}{2 \mu a k}+\alpha \cdot \frac{\mu a k}{2 C}\left(\frac{C\|\mathbf{w}\|_{2}}{\mu a k}+\frac{1}{\sqrt{m}}\right)^{2} \\
& \leq(2 C \alpha+2) \frac{\|\mathbf{w}\|_{2}}{\sqrt{\mu a k}}\left(\left\|\mathbf{x}_{0}\right\|_{1}+\frac{\|\mathbf{w}\|_{2}}{\sqrt{2 \mu}}\right)+\frac{\|\mathbf{w}\|_{2}^{2}}{2 \mu a k}+\alpha \cdot \frac{\mu a k}{2 C}\left(\frac{C\|\mathbf{w}\|_{2}}{\mu a k}+\frac{1}{\sqrt{m}}\right)^{2},
\end{aligned}
$$


which leads to the conclusion. Here, the fourth line is based on

$$
\left\|\mathbf{x}_{T_{01}}^{\#}\right\|_{2} \leq\left\|\mathbf{x}_{T_{01}}^{\#}\right\|_{1} \leq\left\|\mathbf{x}^{\#}\right\|_{1} \leq\left\|\mathbf{x}_{0}\right\|_{1}+\frac{\|\mathbf{w}\|_{2}}{\sqrt{2 \mu}}
$$

where the last inequality follows from (3.6).

We remain to prove (3.8). Note that

$$
\frac{1}{\sqrt{m}}\|\mathcal{A}(H)\|_{2} \geq \frac{1}{m}\|\mathcal{A}(H)\|_{1} \geq \frac{1}{m}\|\mathcal{A}(\bar{H})\|_{1}-\frac{1}{m}\|\mathcal{A}(H-\bar{H})\|_{1},
$$

which implies

$$
\frac{1}{m}\|\mathcal{A}(\bar{H})\|_{1} \leq \frac{1}{m}\|\mathcal{A}(H-\bar{H})\|_{1}+\frac{1}{\sqrt{m}}\|\mathcal{A}(H)\|_{2}
$$

Here we can see that

$$
\frac{1}{m}\|\mathcal{A}(H-\bar{H})\|_{1} \leq \sum_{i=0,1} \frac{1}{m}\left\|\mathcal{A}\left(H_{T_{i}, T_{01}^{c}}+H_{T_{01}^{c}, T_{i}}\right)\right\|_{1}+\frac{1}{m}\left\|\mathcal{A}\left(H_{T_{01}^{c}, T_{01}^{c}}\right)\right\|_{1} .
$$

For $i=0,1$, since $\mathcal{A}(\cdot)$ satisfy the RIP condition of order $(2,2 a k)$ with upper RIP constant $C$, we have

$$
\begin{aligned}
& \frac{1}{m}\left\|\mathcal{A}\left(H_{T_{i}, T_{01}^{c}}+H_{T_{01}^{c}, T_{i}}\right)\right\|_{1}=\frac{1}{m}\left\|\sum_{j \geq 2} \mathcal{A}\left(H_{T_{i}, T_{j}}+H_{T_{j}, T_{i}}\right)\right\|_{1} \\
& \leq \frac{1}{m} \sum_{j \geq 2}\left\|\mathcal{A}\left(H_{T_{i}, T_{j}}+H_{T_{j}, T_{i}}\right)\right\|_{1}=\frac{1}{m} \sum_{j \geq 2}\left\|\mathcal{A}\left(\mathbf{x}_{T_{i}}^{\#}\left(\mathbf{x}_{T_{j}}^{\#}\right)^{*}+\mathbf{x}_{T_{j}}^{\#}\left(\mathbf{x}_{T_{i}}^{\#}\right)^{*}\right)\right\|_{1} \\
& \leq 2 C \sum_{j \geq 2}\left\|\mathbf{x}_{T_{i}}^{\#}\right\|_{2}\left\|\mathbf{x}_{T_{j}}^{\#}\right\|_{2} \leq \frac{2 C}{\sqrt{a k}}\left\|\mathbf{x}_{T_{0}^{c}}^{\#}\right\|_{1}\left\|\mathbf{x}_{T_{i}}^{\#}\right\|_{2} \\
& \leq \frac{2 C}{\sqrt{a}}\|\bar{H}\|_{F}+\frac{2 C\|\mathbf{w}\|_{2}}{\sqrt{2 \mu a k}}\left\|\mathbf{x}_{T_{i}}^{\#}\right\|_{2} .
\end{aligned}
$$

Here, the last line follows from (3.4). On the other hand, based on (3.3), we have

$$
\begin{aligned}
\frac{1}{m}\left\|\mathcal{A}\left(H_{T_{01}^{c}, T_{01}^{c}}\right)\right\|_{1} & \leq \frac{1}{m}\left\|\sum_{i, j \geq 2, i \neq j} \mathcal{A}\left(H_{T_{i}, T_{j}}+H_{T_{j}, T_{i}}\right)\right\|_{1}+\frac{1}{m}\left\|\sum_{i \geq 2} \mathcal{A}\left(H_{T_{i}, T_{i}}\right)\right\|_{1} \\
& \leq C \sum_{i \geq 2, j \geq 2}\left\|H_{T_{i}, T_{j}}\right\|_{F} \leq \frac{C}{a}\|\bar{H}\|_{F}+\frac{C\|\mathbf{w}\|_{2}}{\mu a k}\|\mathcal{A}(H)\|_{2}-\frac{C}{2 \mu a k}\|\mathcal{A}(H)\|_{2}^{2} .
\end{aligned}
$$


As $\mathcal{A}(\cdot)$ satisfy the RIP condition of order $(2,2 a k)$ with lower RIP constant $c>0$, combining (3.9), (3.10) and (3.11), we obtain that

$$
\begin{aligned}
& c\|\bar{H}\|_{F} \leq \frac{1}{m}\|\mathcal{A}(\bar{H})\|_{1} \leq \frac{1}{\sqrt{m}}\|\mathcal{A}(H)\|_{2}+\frac{1}{m}\|\mathcal{A}(H-\bar{H})\|_{1} \\
& \leq \frac{1}{\sqrt{m}}\|\mathcal{A}(H)\|_{2}+\sum_{i=0,1} \frac{1}{m}\left\|\mathcal{A}\left(H_{T_{i}, T_{01}^{c}}+H_{T_{01}^{c}, T_{i}}\right)\right\|_{1}+\frac{1}{m}\left\|\mathcal{A}\left(H_{T_{01}^{c}, T_{01}^{c}}\right)\right\|_{1} \\
& \leq \frac{1}{\sqrt{m}}\|\mathcal{A}(H)\|_{2}+2 C \frac{\|\mathbf{w}\|_{2}}{\sqrt{\mu a k}}\left(\left\|\mathbf{x}_{T_{0}}^{\#}\right\|_{2}+\left\|\mathbf{x}_{T_{1}}^{\#}\right\|_{2}\right)+\left(\frac{C}{a}+\frac{4 \sqrt{2} C}{\sqrt{a}}\right)\|\bar{H}\|_{F}+\frac{C\|\mathbf{w}\|_{2}}{\mu a k}\|\mathcal{A}(H)\|_{2}-\frac{C}{2 \mu a k}\|\mathcal{A}(H)\|_{2}^{2} \\
& \leq \frac{1}{\sqrt{m}}\|\mathcal{A}(H)\|_{2}+2 C \frac{\|\mathbf{w}\|_{2}}{\sqrt{\mu a k}}\left\|\mathbf{x}_{T_{01}}^{\#}\right\|_{2}+\left(\frac{C}{a}+\frac{4 \sqrt{2} C}{\sqrt{a}}\right)\|\bar{H}\|_{F}+\frac{C\|\mathbf{w}\|_{2}}{\mu a k}\|\mathcal{A}(H)\|_{2}-\frac{C}{2 \mu a k}\|\mathcal{A}(H)\|_{2}^{2},
\end{aligned}
$$

which implies

$$
\begin{aligned}
\left(c-\frac{C}{a}-\frac{4 \sqrt{2} C}{\sqrt{a}}\right)\|\bar{H}\|_{F} & \leq 2 C \frac{\|\mathbf{w}\|_{2}}{\sqrt{\mu a k}}\left\|\mathbf{x}_{T_{01}}^{\#}\right\|_{2}+\left(\frac{C\|\mathbf{w}\|_{2}}{\mu a k}+\frac{1}{\sqrt{m}}\right)\|\mathcal{A}(H)\|_{2}-\frac{C}{2 \mu a k}\|\mathcal{A}(H)\|_{2}^{2} \\
& \leq 2 C \frac{\|\mathbf{w}\|_{2}}{\sqrt{\mu a k}}\left\|\mathbf{x}_{T_{01}}^{\#}\right\|_{2}+\frac{\mu a k}{2 C}\left(\frac{C\|\mathbf{w}\|_{2}}{\mu a k}+\frac{1}{\sqrt{m}}\right)^{2} .
\end{aligned}
$$

It leads to the inequality (3.8).

\section{Proof of Lemma 1.1}

Denote $\mathbb{R}_{\text {sym }}^{d \times d}$ as the set of symmetric real $d \times d$ matrices, and $\mathbb{R}_{\text {skew }}^{d \times d}$ as the set of skew-symmetric real $d \times d$ matrices. If $X \in \mathbb{H}^{d \times d}$, then $X$ can be written as $X=X_{1}+i X_{2}$, where $X_{1} \in \mathbb{R}_{\mathrm{sym}}^{d \times d}$ and $X_{2} \in \mathbb{R}_{\text {skew }}^{d \times d}$ are the real and imaginary parts of $X$. Thus the set $\left\{X \in \mathbb{H}^{d \times d}: X \succeq 0\right\}$ corresponds to

$\mathbb{H}_{+}^{d \times d}:=\left\{\left[\begin{array}{l}X_{1} \\ X_{2}\end{array}\right]:\left(X_{1}, X_{2}\right) \in \mathbb{R}_{\text {sym }}^{d \times d} \times \mathbb{R}_{\text {skew }}^{d \times d}, \mathbf{z}_{1}^{\mathrm{T}} X_{1} \mathbf{z}_{1}+\mathbf{z}_{2}^{\mathrm{T}} X_{1} \mathbf{z}_{2}+\mathbf{z}_{2}^{\mathrm{T}} X_{2} \mathbf{z}_{1}-\mathbf{z}_{1}^{\mathrm{T}} X_{2} \mathbf{z}_{2} \geq 0\right.$ for all $\left.\mathbf{z}_{1}, \mathbf{z}_{2} \in \mathbb{R}^{d}\right\}$.

Let $\widetilde{\mathcal{A}}: \mathbb{R}^{2 d \times d} \rightarrow \mathbb{R}^{m}$ be defined by

$$
\left[\begin{array}{l}
X_{1} \\
X_{2}
\end{array}\right] \mapsto\left(\mathscr{R}\left(\mathbf{a}_{i}\right)^{\mathrm{T}} X_{1} \mathscr{R}\left(\mathbf{a}_{i}\right)+\mathscr{I}\left(\mathbf{a}_{i}\right)^{\mathrm{T}} X_{1} \mathscr{I}\left(\mathbf{a}_{i}\right)+\mathscr{I}\left(\mathbf{a}_{i}\right)^{\mathrm{T}} X_{2} \mathscr{R}\left(\mathbf{a}_{i}\right)-\mathscr{R}\left(\mathbf{a}_{i}\right)^{\mathrm{T}} X_{2} \mathscr{I}\left(\mathbf{a}_{i}\right)\right)_{i=1}^{m}
$$

Then $\mathcal{A}(X)=\widetilde{A}\left(\left[\begin{array}{l}X_{1} \\ X_{2}\end{array}\right]\right)$. By a simple calculation, its conjugate map $\widetilde{\mathcal{A}}^{*}: \mathbb{R}^{m} \rightarrow \mathbb{R}^{2 d \times d}$ is given by

$$
\left(b_{i}\right)_{i=1}^{m} \mapsto\left[\begin{array}{l}
\sum_{i=1}^{m} b_{i}\left(\mathscr{R}\left(\mathbf{a}_{i}\right) \mathscr{R}\left(\mathbf{a}_{i}\right)^{\mathrm{T}}+\mathscr{I}\left(\mathbf{a}_{i}\right) \mathscr{I}\left(\mathbf{a}_{i}\right)^{\mathrm{T}}\right) \\
\sum_{i=1}^{m} b_{i}\left(\mathscr{I}\left(\mathbf{a}_{i}\right) \mathscr{R}\left(\mathbf{a}_{i}\right)^{\mathrm{T}}-\mathscr{R}\left(\mathbf{a}_{i}\right) \mathscr{I}\left(\mathbf{a}_{i}\right)^{\mathrm{T}}\right)
\end{array}\right] .
$$

For $X=X_{1}+i X_{2} \in \mathbb{C}^{d \times d},\|X\|_{1}$ and $\|X\|_{F}$ can also be written as

$$
\|X\|_{1}=\left\|\left[\begin{array}{c}
\operatorname{vec}\left(X_{1}\right) \\
\operatorname{vec}\left(X_{2}\right)
\end{array}\right]\right\|_{1,2}=\sum_{i, j} \sqrt{\left[X_{1}\right]_{i, j}^{2}+\left[X_{2}\right]_{i, j}^{2}}, \quad \text { and } \quad\|X\|_{F}=\left\|\left[\begin{array}{c}
X_{1} \\
X_{2}
\end{array}\right]\right\|_{F} .
$$

Using the notations above, we recast the model (1.8) as follows.

$$
\min _{X_{1}, X_{2}} \lambda\left(\operatorname{Tr}\left(X_{1}\right)-\left\|\left[\begin{array}{l}
X_{1} \\
X_{2}
\end{array}\right]\right\|_{F}\right)+\mu\left\|\left[\begin{array}{l}
\operatorname{vec}\left(X_{1}\right) \\
\operatorname{vec}\left(X_{2}\right)
\end{array}\right]\right\|_{1,2}+\frac{1}{2}\left\|\widetilde{\mathcal{A}}\left(\left[\begin{array}{c}
X_{1} \\
X_{2}
\end{array}\right]\right)-\mathbf{b}\right\|_{2}^{2} \quad \text { s.t. }\left[\begin{array}{c}
X_{1} \\
X_{2}
\end{array}\right] \in \mathbb{H}_{+}^{d \times d} .
$$


If $\left(X_{1}^{\#}, X_{2}^{\#}\right)$ is a minimizer of [4.3), then the optimal solution $X^{\#}$ of (1.8) satisfies $X^{\#}=X_{1}^{\#}+i X_{2}^{\#}$.

In order to prove Lemma 1.1, we first introduce some technical lemmas in convex optimization and matrix theory. Assume that $\Omega \subset \mathbb{R}^{n}$. We use $T_{\Omega}\left(\mathbf{x}^{\#}\right)$ and $T_{\Omega}\left(\mathbf{x}^{\#}\right)^{*}$ to denote the tangent cone of $\Omega$ at $\mathbf{x}^{\#} \in \Omega$ and its dual cone, respectively. Particularly, we have

Proposition 4.1. If $\Omega$ is a convex cone in $\mathbb{R}^{n}$ and $\mathbf{x}^{\#} \in \Omega$, then

$$
T_{\Omega}\left(\mathbf{x}^{\#}\right)^{*}=\left\{\mathbf{y} \in \mathbb{R}^{n}:\langle\mathbf{y}, \mathbf{x}\rangle \leq 0 \text { for all } \mathbf{x} \in \Omega \text {, and }\left\langle\mathbf{y}, \mathbf{x}^{\#}\right\rangle=0\right\} \text {. }
$$

Proof. According to Proposition 4.6.3 in [2], we have

$$
T_{\Omega}\left(\mathbf{x}^{\#}\right)^{*}=\left\{\mathbf{y} \in \mathbb{R}^{n}:\left\langle\mathbf{y}, \mathbf{x}-\mathbf{x}^{\#}\right\rangle \leq 0 \text { for all } \mathbf{x} \in \Omega\right\} .
$$

Assume that $\mathbf{y} \in T_{\Omega}\left(\mathbf{x}^{\#}\right)^{*}$. Then $\left\langle\mathbf{y}, \mathbf{x}-\mathbf{x}^{\#}\right\rangle \leq 0$ for all $\mathbf{x} \in \Omega$. Since $\Omega$ is a cone, we have $\mathbf{x}^{\#} / 2,2 \mathbf{x}^{\#} \in \Omega$. Taking $\mathbf{x}=2 \mathbf{x}^{\#}$, we obtain $\left\langle\mathbf{y}, \mathbf{x}^{\#}\right\rangle \leq 0$. Similarly, taking $\mathbf{x}=\mathbf{x}^{\#} / 2$, we have $\left\langle\mathbf{y}, \mathbf{x}^{\#}\right\rangle \geq 0$. We arrive at $\left\langle\mathbf{y}, \mathbf{x}^{\#}\right\rangle=0$, which leads to $\langle\mathbf{y}, \mathbf{x}\rangle \leq 0$ for all $\mathbf{x} \in \Omega$.

The following theorem provides some properties of local minimum on constrained model.

Proposition 4.2. 2, Proposition 4.7.3] Let $\mathbf{x}^{\#}$ be a local minimizer of the model:

$$
\min _{\mathbf{x} \in \Omega} f_{1}(\mathbf{x})+f_{2}(\mathbf{x})
$$

where $f_{1}$ is convex and $f_{2}$ is smooth over a subset $\Omega$ of $\mathbb{R}^{n}$. Assume that the tangent cone $T_{\Omega}\left(\mathbf{x}^{\#}\right)$ is convex. Then

$$
-\nabla f_{2}\left(\mathbf{x}^{\#}\right) \in \partial f_{1}\left(\mathbf{x}^{\#}\right)+T_{\Omega}\left(\mathbf{x}^{\#}\right)^{*} .
$$

We next present the sub-gradient set of $\partial\left(\|X\|_{1}\right)$ :

Proposition 4.3. (1]) Assume that $X=X_{1}+i X_{2}$ with $X_{1}, X_{2} \in \mathbb{R}^{d \times d}$. Then the subgradient set of $\|X\|_{1}$ in real space is

$$
\begin{array}{r}
\partial\left(\left\|\left[\begin{array}{l}
\operatorname{vec}\left(X_{1}\right) \\
\operatorname{vec}\left(X_{2}\right)
\end{array}\right]\right\|_{1,2}\right):=\left\{\left[\begin{array}{l}
G_{1} \\
G_{2}
\end{array}\right], G_{1}, G_{2} \in \mathbb{R}^{d \times d}:\left[G_{1}\right]_{i_{1}, i_{2}}^{2}+\left[G_{2}\right]_{i_{1}, i_{2}}^{2} \leq 1, \text { if }\left[X_{1}\right]_{i_{1}, i_{2}}=\left[X_{2}\right]_{i_{1}, i_{2}}=0 ;\right. \\
\left.\left(\left[G_{1}\right]_{i_{1}, i_{2}},\left[G_{2}\right]_{i_{1}, i_{2}}\right)=\frac{\left(\left[X_{1}\right]_{i_{1}, i_{2}},\left[X_{2}\right]_{i_{1}, i_{2}}\right)}{\sqrt{\left[X_{1}\right]_{i_{1}, i_{2}}^{2}+\left[X_{2}\right]_{i_{1}, i_{2}}^{2}}}, \text { otherwise }\right\}
\end{array}
$$

Combining Proposition 4.1 Proposition 4.2 with Proposition 4.3 we have

Lemma 4.4. Assume that $\left(X_{1}^{\#}, X_{2}^{\#}\right)$ is a local minimizer of model 4.3). Then there exist $\left[\begin{array}{l}\Lambda_{1} \\ \Lambda_{2}\end{array}\right] \in$ $\mathbb{H}_{+}^{d \times d}$ and $\left[\begin{array}{l}G_{1} \\ G_{2}\end{array}\right] \in \partial\left(\left\|\left[\begin{array}{l}\operatorname{vec}\left(X_{1}^{\#}\right) \\ \operatorname{vec}\left(X_{2}^{\#}\right)\end{array}\right]\right\|_{1,2}\right)$ such that the followings hold:

(i) Stationary condition:

$$
\lambda\left(\left[\begin{array}{l}
\mathbf{I} \\
\mathbf{0}
\end{array}\right]-\left[\begin{array}{c}
X_{1}^{\#} \\
X_{2}^{\#}
\end{array}\right] /\left\|\left[\begin{array}{c}
X_{1}^{\#} \\
X_{2}^{\#}
\end{array}\right]\right\|_{F}\right)+\mu\left[\begin{array}{l}
G_{1} \\
G_{2}
\end{array}\right]+\widetilde{\mathcal{A}}^{*}\left(\widetilde{\mathcal{A}}\left(\left[\begin{array}{c}
X_{1}^{\#} \\
X_{2}^{\#}
\end{array}\right]\right)-\mathbf{b}\right)-\left[\begin{array}{c}
\Lambda_{1} \\
\Lambda_{2}
\end{array}\right]=0
$$

(ii) Complementary slackness condition:

$$
\left\langle\left[\begin{array}{l}
\Lambda_{1} \\
\Lambda_{2}
\end{array}\right],\left[\begin{array}{l}
X_{1}^{\#} \\
X_{2}^{\#}
\end{array}\right]\right\rangle=0 .
$$


Proof. Set

$$
\begin{aligned}
& f_{1}\left(X_{1}, X_{2}\right):=\mu\left\|\left[\begin{array}{c}
\operatorname{vec}\left(X_{1}\right) \\
\operatorname{vec}\left(X_{2}\right)
\end{array}\right]\right\|_{1,2}, \\
& f_{2}\left(X_{1}, X_{2}\right):=\lambda\left(\operatorname{Tr}\left(X_{1}\right)-\left\|\left[\begin{array}{l}
X_{1} \\
X_{2}
\end{array}\right]\right\|_{F}\right)+\frac{1}{2}\left\|\widetilde{\mathcal{A}}\left(\left[\begin{array}{l}
X_{1} \\
X_{2}
\end{array}\right]\right)-\mathbf{b}\right\|_{2}^{2},
\end{aligned}
$$

and $\Omega:=\mathbb{H}_{+}^{d \times d}$. Then $f_{1}$ is convex and $f_{2}$ is smooth. Since $\Omega$ is convex, we obtain that $T_{\Omega}\left(\left[\begin{array}{c}X_{1}^{\#} \\ X_{2}^{\#}\end{array}\right]\right)$ is convex by Proposition 4.6.2 in [2]. According to Proposition 4.2, there exists $-\left[\begin{array}{l}\Lambda_{1} \\ \Lambda_{2}\end{array}\right] \in T_{\Omega}\left(\left[\begin{array}{c}X_{1}^{\#} \\ X_{2}^{\#}\end{array}\right]\right)^{*}$ such that the stationary condition (4.6) holds. Furthermore, we can use Proposition 4.1 to obtain the complementary slackness condition (4.7).

We remain to prove $\left[\begin{array}{l}\Lambda_{1} \\ \Lambda_{2}\end{array}\right] \in \mathbb{H}_{+}^{d \times d}$. Take $T_{1}=\mathbf{t}_{1} \mathbf{t}_{1}^{\mathrm{T}}+\mathbf{t}_{2} \mathbf{t}_{2}^{\mathrm{T}}$ and $T_{2}=\mathbf{t}_{2} \mathbf{t}_{1}^{\mathrm{T}}-\mathbf{t}_{1} \mathbf{t}_{2}^{\mathrm{T}}$ for any fixed $\mathbf{t}_{1}, \mathbf{t}_{2} \in \mathbb{R}^{d}$. Then $\left[\begin{array}{l}T_{1} \\ T_{2}\end{array}\right] \in \Omega$. By the definition of $T_{\Omega}\left(\left[\begin{array}{c}X_{1}^{\#} \\ X_{2}^{\#}\end{array}\right]\right)^{*}$ and Proposition 4.2 , we obtain that

$$
\left\langle-\left[\begin{array}{l}
\Lambda_{1} \\
\Lambda_{2}
\end{array}\right],\left[\begin{array}{l}
T_{1} \\
T_{2}
\end{array}\right]\right\rangle \leq 0
$$

which implies

$$
\mathbf{t}_{1}^{\mathrm{T}} \Lambda_{1} \mathbf{t}_{1}+\mathbf{t}_{2}^{\mathrm{T}} \Lambda_{1} \mathbf{t}_{2}+\mathbf{t}_{2}^{\mathrm{T}} \Lambda_{2} \mathbf{t}_{1}-\mathbf{t}_{1}^{\mathrm{T}} \Lambda_{2} \mathbf{t}_{2} \geq 0 \text { for any } \mathbf{t}_{1}, \mathbf{t}_{2} \in \mathbb{R}^{d} .
$$

If $\left(\Lambda_{1}, \Lambda_{2}\right) \in \mathbb{R}_{\mathrm{sym}}^{d \times d} \times \mathbb{R}_{\mathrm{skew}}^{d \times d}$, then we arrive at the conclusion. Otherwise, we can replace $\Lambda_{1}$ and $\Lambda_{2}$ by

$$
\widetilde{\Lambda}_{1}:=\frac{\Lambda_{1}+\Lambda_{1}^{\mathrm{T}}}{2} \text { and } \widetilde{\Lambda}_{2}:=\frac{\Lambda_{2}-\Lambda_{2}^{\mathrm{T}}}{2} .
$$

Noting that $\left(\widetilde{\Lambda}_{1}, \widetilde{\Lambda}_{2}\right) \in \mathbb{R}_{\text {sym }}^{d \times d} \times \mathbb{R}_{\text {skew }}^{d \times d}$ and

$$
\left\langle-\left[\begin{array}{c}
\widetilde{\Lambda}_{1} \\
\widetilde{\Lambda}_{2}
\end{array}\right],\left[\begin{array}{l}
T_{1} \\
T_{2}
\end{array}\right]\right\rangle=\left\langle-\left[\begin{array}{l}
\Lambda_{1} \\
\Lambda_{2}
\end{array}\right],\left[\begin{array}{l}
T_{1} \\
T_{2}
\end{array}\right]\right\rangle \leq 0
$$

we obtain that $\left[\begin{array}{l}\widetilde{\Lambda}_{1} \\ \widetilde{\Lambda}_{2}\end{array}\right] \in \mathbb{H}_{+}^{d \times d}$. After a simple calculation, we also have

$$
\lambda\left(\left[\begin{array}{l}
\mathbf{I} \\
\mathbf{0}
\end{array}\right]-\left[\begin{array}{c}
X_{1}^{\#} \\
X_{2}^{\#}
\end{array}\right] /\left\|\left[\begin{array}{l}
X_{1}^{\#} \\
X_{2}^{\#}
\end{array}\right]\right\|_{F}\right)+\mu\left[\begin{array}{l}
\widetilde{G}_{1} \\
\widetilde{G}_{2}
\end{array}\right]+\widetilde{\mathcal{A}}^{*}\left(\widetilde{\mathcal{A}}\left(\left[\begin{array}{c}
X_{1}^{\#} \\
X_{2}^{\#}
\end{array}\right]\right)-\mathbf{b}\right)-\left[\begin{array}{l}
\widetilde{\Lambda}_{1} \\
\widetilde{\Lambda}_{2}
\end{array}\right]=0
$$

and

$$
\left\langle\left[\begin{array}{c}
\widetilde{\Lambda}_{1} \\
\widetilde{\Lambda}_{2}
\end{array}\right],\left[\begin{array}{c}
X_{1}^{\#} \\
X_{2}^{\#}
\end{array}\right]\right\rangle=\left\langle\left[\begin{array}{c}
\Lambda_{1} \\
\Lambda_{2}
\end{array}\right],\left[\begin{array}{c}
X_{1}^{\#} \\
X_{2}^{\#}
\end{array}\right]\right\rangle=0
$$

where $\widetilde{G}_{1}:=\frac{G_{1}+G_{1}^{\mathrm{T}}}{2}, \widetilde{G}_{2}:=\frac{G_{2}-G_{2}^{\mathrm{T}}}{2}$ and

$$
\left[\begin{array}{l}
\widetilde{G}_{1} \\
\widetilde{G}_{2}
\end{array}\right] \in \partial\left(\left\|\left[\begin{array}{l}
\operatorname{vec}\left(X_{1}^{\#}\right) \\
\operatorname{vec}\left(X_{2}^{\#}\right)
\end{array}\right]\right\|_{1,2}\right) .
$$


Therefore, the stationary condition (4.6) and complementary slackness condition (4.7) also hold for $\left[\begin{array}{l}\Lambda_{1} \\ \Lambda_{2}\end{array}\right]:=\left[\begin{array}{l}\widetilde{\Lambda}_{1} \\ \widetilde{\Lambda}_{2}\end{array}\right]$

We next present the proof of Lemma 1.1

Proof of Lemma 1.1. Since $\frac{1}{2}\|\mathbf{b}\|_{2}^{2}>\mu\left\|\mathbf{x}_{0}\right\|_{1}^{2}+\frac{1}{2}\|\mathbf{w}\|_{2}^{2}$, we obtain that $X^{\#} \neq 0$.

We next consider the equivalent model (4.3) with global minimizer $\left(X_{1}^{\#}, X_{2}^{\#}\right)$. According to Lemma 4.4 there exist $\left[\begin{array}{l}\Lambda_{1} \\ \Lambda_{2}\end{array}\right] \in \mathbb{H}_{+}^{d \times d}$ and $\left[\begin{array}{l}G_{1} \\ G_{2}\end{array}\right] \in \partial\left(\left\|\left[\begin{array}{l}\operatorname{vec}\left(X_{1}^{\#}\right) \\ \operatorname{vec}\left(X_{2}^{\#}\right)\end{array}\right]\right\|_{1,2}\right)$ such that the following holds:

$$
\lambda\left(\left[\begin{array}{l}
\mathbf{I} \\
\mathbf{0}
\end{array}\right]-\left[\begin{array}{c}
X_{1}^{\#} \\
X_{2}^{\#}
\end{array}\right] /\left\|\left[\begin{array}{l}
X_{1}^{\#} \\
X_{2}^{\#}
\end{array}\right]\right\|_{F}\right)+\mu\left[\begin{array}{l}
G_{1} \\
G_{2}
\end{array}\right]+\widetilde{\mathcal{A}}^{*}\left(\widetilde{\mathcal{A}}\left(\left[\begin{array}{c}
X_{1}^{\#} \\
X_{2}^{\#}
\end{array}\right]\right)-\mathbf{b}\right)-\left[\begin{array}{l}
\Lambda_{1} \\
\Lambda_{2}
\end{array}\right]=0
$$

and

$$
\left\langle\left[\begin{array}{l}
\Lambda_{1} \\
\Lambda_{2}
\end{array}\right],\left[\begin{array}{c}
X_{1}^{\#} \\
X_{2}^{\#}
\end{array}\right]\right\rangle=0
$$

According to (4.9), we obtain that

$$
\begin{aligned}
\left\|\widetilde{\mathcal{A}}^{*}\left(\widetilde{\mathcal{A}}\left(\left[\begin{array}{c}
X_{1}^{\#} \\
X_{2}^{\#}
\end{array}\right]\right)-\mathbf{b}\right)+\mu\left[\begin{array}{c}
G_{1} \\
G_{2}
\end{array}\right]\right\|_{F} & =\left\|\lambda\left(\left[\begin{array}{l}
\mathbf{I} \\
\mathbf{0}
\end{array}\right]-\left[\begin{array}{c}
X_{1}^{\#} \\
X_{2}^{\#}
\end{array}\right] /\left\|\left[\begin{array}{c}
X_{1}^{\#} \\
X_{2}^{\#}
\end{array}\right]\right\|_{F}\right)-\left[\begin{array}{c}
\Lambda_{1} \\
\Lambda_{2}
\end{array}\right]\right\|_{F} \\
& =\left\|\lambda\left(\mathbf{I}-\frac{X^{\#}}{\left\|X^{\#}\right\|_{F}}\right)-\Lambda\right\|_{F} \\
& \geq \lambda(\sqrt{r}-1),
\end{aligned}
$$

where $\Lambda:=\Lambda_{1}+i \Lambda_{2} \in \mathbb{C}^{d \times d}$ and $r:=\operatorname{rank}\left(X^{\#}\right)$. The last inequality in (4.11) follows from (4.10) and Lemma 2.3

On the other hand, we have

$$
\begin{aligned}
\left\|\widetilde{\mathcal{A}}^{*}\left(\widetilde{\mathcal{A}}\left(\left[\begin{array}{c}
X_{1}^{\#} \\
X_{2}^{\#}
\end{array}\right]\right)-\mathbf{b}\right)+\mu\left[\begin{array}{c}
G_{1} \\
G_{2}
\end{array}\right]\right\|_{F} & \leq\left\|\widetilde{\mathcal{A}}^{*}\left(\widetilde{\mathcal{A}}\left(\left[\begin{array}{c}
X_{1}^{\#} \\
X_{2}^{\#}
\end{array}\right]\right)-\mathbf{b}\right)\right\|_{F}+\mu\left\|\left[\begin{array}{l}
G_{1} \\
G_{2}
\end{array}\right]\right\|_{F} \\
& \leq\|\mathcal{A}\|\left\|\widetilde{\mathcal{A}}\left(\left[\begin{array}{c}
X_{1}^{\#} \\
X_{2}^{\#}
\end{array}\right]\right)-\mathbf{b}\right\|_{2}+\mu d \\
& =\|\mathcal{A}\|\left\|\mathcal{A}\left(X^{\#}\right)-\mathbf{b}\right\|_{2}+\mu d \\
& \leq\|\mathcal{A}\| \sqrt{2 \mu\left\|\mathbf{x}_{0}\right\|_{1}^{2}+\|\mathbf{w}\|_{2}^{2}}+\mu d \\
& \leq\|\mathcal{A}\|\left(\sqrt{2 \mu}\left\|\mathbf{x}_{0}\right\|_{1}+\|\mathbf{w}\|_{2}\right)+\mu d .
\end{aligned}
$$

Here, the second inequality follows from Proposition 4.3 and $\left[G_{1}\right]_{i_{1}, i_{2}}^{2}+\left[G_{1}\right]_{i_{1}, i_{2}}^{2} \leq 1$ for any $i_{1}, i_{2} \in$ $\{1, \ldots, d\}$. Combing (4.11) and (4.12), we obtain that

$$
\lambda(\sqrt{r}-1) \leq \mu d+\|\mathcal{A}\|\left(\sqrt{2 \mu}\left\|\mathbf{x}_{0}\right\|_{1}+\|\mathbf{w}\|_{2}\right) .
$$

By the assumption on $\lambda$ in (1.9) as

$$
\lambda>\frac{\mu d+\|\mathcal{A}\|\left(\sqrt{2 \mu}\left\|\mathbf{x}_{0}\right\|_{1}+\|\mathbf{w}\|_{2}\right)}{\sqrt{2}-1},
$$




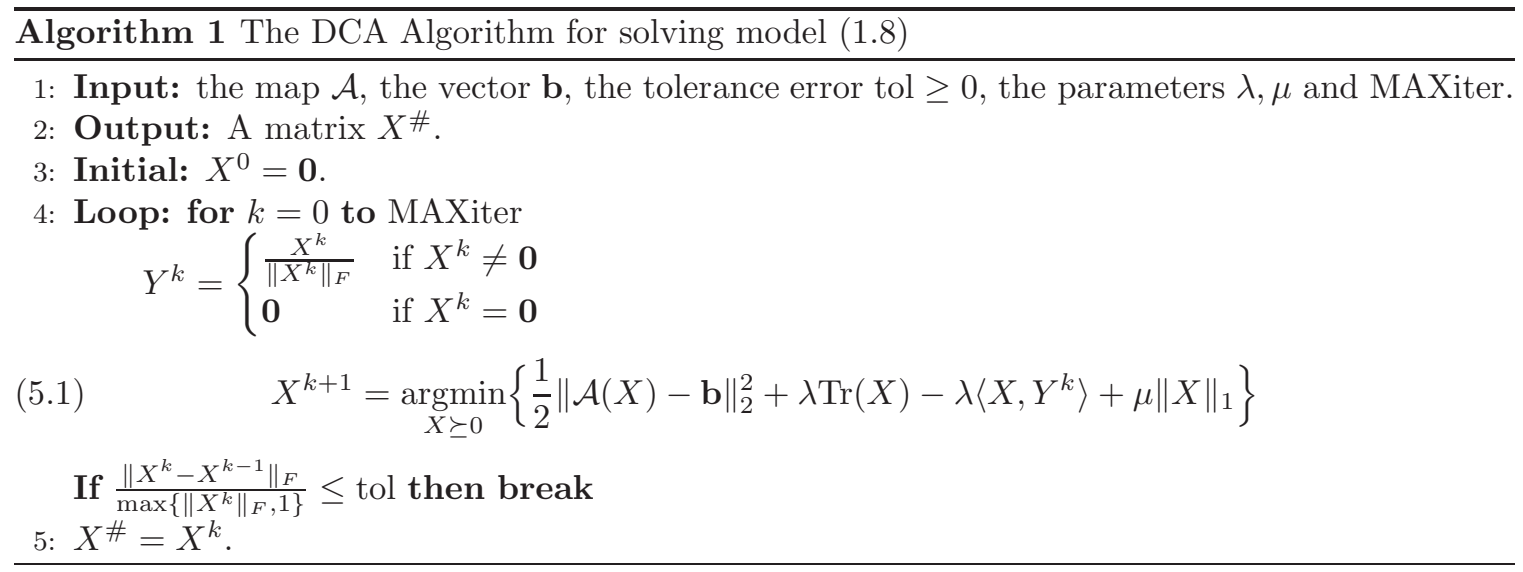

we have

$$
\frac{\sqrt{r}-1}{\sqrt{2}-1}\left(\mu d+\|\mathcal{A}\|\left(\sqrt{2 \mu}\left\|\mathbf{x}_{0}\right\|_{1}+\|\mathbf{w}\|_{2}\right)\right) \leq \mu d+\|\mathcal{A}\|\left(\sqrt{2 \mu}\left\|\mathbf{x}_{0}\right\|_{1}+\|\mathbf{w}\|_{2}\right) .
$$

Thus the only proper choice of $r$ is $r=1$.

\section{Algorithms for solving Sparse PhaseliftOff}

5.1. The DCA algorithm. In this section, we establish an algorithm to solve the Sparse PhaseLiftOff model (1.8), which is stated in Algorithm 1 . Our algorithm is based on DCA, which is a descent method introduced by Tao and An [14, 15. DCA is also studied in compressed sensing, and in matrix recovery problem (see 21, 23, 24).

The step 6 of Algorithm 1 is to solve a subproblem (5.1). We suggest employing ADMM method [3] to solve it, which is shown in Algorithm 2. The convergence rate of ADMM was established in [9]. To derive ADMM, we rewrite (5.1) as

$$
\min _{X_{3} \succeq 0, X_{3}=X_{1}, X_{3}=X_{2}} \frac{1}{2}\left\|\mathcal{A}\left(X_{1}\right)-\mathbf{b}\right\|_{2}^{2}+\lambda \operatorname{Tr}\left(X_{1}\right)-\lambda\left\langle X_{1}, Y^{k}\right\rangle+\mu\left\|X_{2}\right\|_{1} .
$$

The problem (5.2) is called global consensus problem [3, Equation (7.2)] with local variables $X_{1}$ and $X_{2}$ and a common global variable $X_{3}$. The augmented Lagrangian function corresponding to (5.2) is

$$
\begin{aligned}
\mathcal{L}_{\delta}\left(X_{1}, X_{2}, X_{3}, Y_{1}, Y_{2}\right)= & \frac{1}{2}\left\|\mathcal{A}\left(X_{1}\right)-\mathbf{b}\right\|_{2}^{2}+\left\langle X_{1}, \lambda\left(\mathbf{I}-Y^{k}\right)\right\rangle+\mu\left\|X_{2}\right\|_{1}+g_{\succeq}\left(X_{3}\right) \\
& +\left\langle Y_{1}, X_{1}-X_{3}\right\rangle+\left\langle Y_{2}, X_{2}-X_{3}\right\rangle+\frac{\delta}{2}\left\|X_{1}-X_{3}\right\|_{F}^{2}+\frac{\delta}{2}\left\|X_{2}-X_{3}\right\|_{F}^{2},
\end{aligned}
$$

where $Y_{1}, Y_{2}$ are dual variables, $\delta$ is augmented Lagrangian parameter and

$$
g_{\succeq 0}(Z)= \begin{cases}0 & \text { if } Z \succeq 0, \\ \infty & \text { otherwise. }\end{cases}
$$

We can employ the standard ADMM to solve

$$
\min _{X_{1}, X_{2}, X_{3}, Y_{1}, Y_{2}} \mathcal{L}_{\delta}\left(X_{1}, X_{2}, X_{3}, Y_{1}, Y_{2}\right),
$$




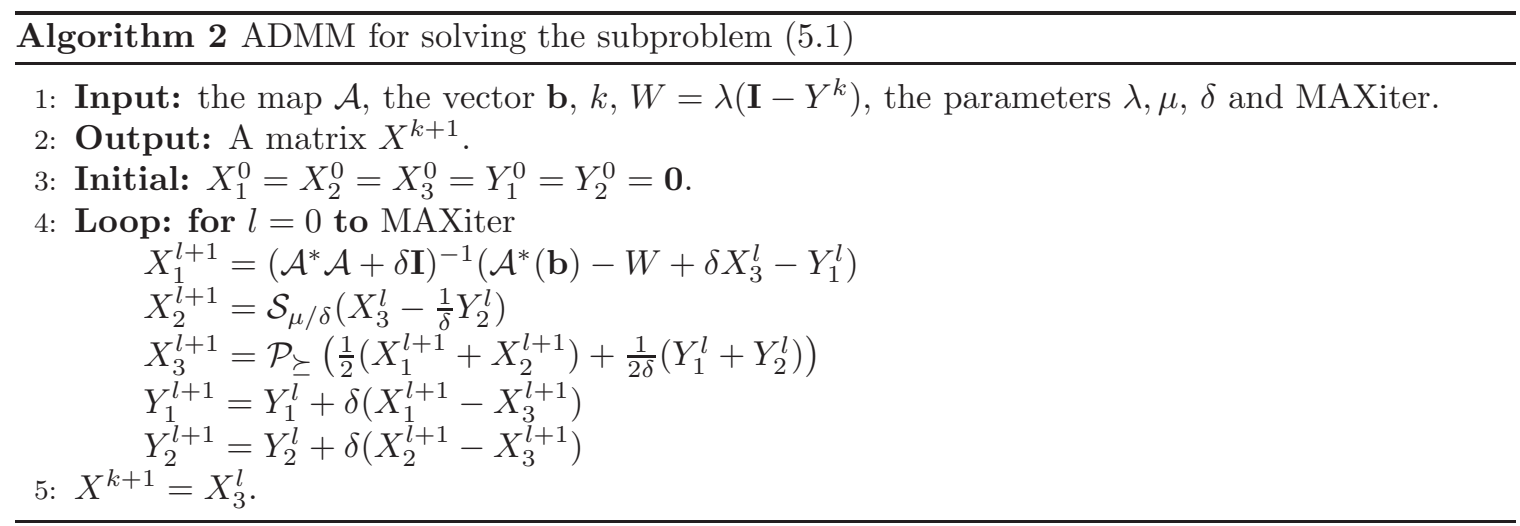

which consists of updating on both the primal and dual variables [3, Equation (7.3)-Equation (7.5)]:

$$
\left\{\begin{array}{l}
X_{1}^{l+1}=\arg \min _{X_{1}} \mathcal{L}_{\delta}\left(X_{1}, X_{2}^{l}, X_{3}^{l}, Y_{1}^{l}, Y_{2}^{l}\right) \\
X_{2}^{l+1}=\arg \min _{X_{2}} \mathcal{L}_{\delta}\left(X_{1}^{l+1}, X_{2}, X_{3}^{l}, Y_{1}^{l}, Y_{2}^{l}\right) \\
X_{3}^{l+1}=\arg \min _{X_{3}} \mathcal{L}_{\delta}\left(X_{1}^{l+1}, X_{2}^{l+1}, X_{3}, Y_{1}^{l}, Y_{2}^{l}\right) \\
Y_{1}^{l+1}=Y_{1}^{l}+\delta\left(X_{1}^{l+1}-X_{3}^{l+1}\right) \\
Y_{2}^{l+1}=Y_{2}^{l}+\delta\left(X_{2}^{l+1}-X_{3}^{l+1}\right)
\end{array}\right.
$$

According to $[3], \delta$ can be fixed or adaptively updated following the rules below:

$$
\delta^{l+1}= \begin{cases}2 \delta^{l} & \text { if }\left\|R^{l}\right\|_{F}>10\left\|S^{l}\right\|_{F} \\ \delta^{l} / 2 & \text { if }\left\|R^{l}\right\|_{F}<\frac{1}{10}\left\|S^{l}\right\|_{F}, \\ \delta^{l} & \text { otherwise }\end{cases}
$$

where $\left\|R^{l}\right\|_{F}^{2}=\left\|X_{1}^{l}-X_{3}^{l}\right\|_{F}^{2}+\left\|X_{2}^{l}-X_{3}^{l}\right\|_{F}^{2}$, and $\left\|S^{l}\right\|_{F}^{2}=2\left(\delta^{l}\right)^{2}\left\|X_{3}^{l}-X_{3}^{l-1}\right\|_{F}^{2}$.

More explicitly, we state ADMM algorithm for solving (5.4) in Algorithm 2. In Algorithm 2, we use $\mathcal{S}_{\lambda}: \mathbb{C}^{n \times n} \rightarrow \mathbb{C}^{n \times n}$ to denote the soft-thresholding operator on each elements of the matrix, i.e.,

$$
\left[\mathcal{S}_{\lambda}(Z)\right]_{i, j}= \begin{cases}\left(\left|Z_{i, j}\right|-\lambda\right) \frac{Z_{i, j}}{\left|Z_{i, j}\right|} & \left|Z_{i, j}\right| \geq \lambda, \\ 0 & \text { otherwise }\end{cases}
$$

We use $\mathcal{P}_{\succeq}: \mathbb{H}^{n \times n} \rightarrow \mathbb{H}^{n \times n}$ to denote the projection on the the positive semidefinite cone, i.e.,

$$
\mathcal{P}_{\succeq}(X)=U \max \{\Sigma, \mathbf{0}\} U^{*},
$$

where $X=U \Sigma U^{*}$ is the eigenvalue decomposition of $X$.

5.2. The Convergence property of Algorithm 1 The aim of this subsection is to study the convergence property of Algorithm 11 Motivated by the techniques developed in [23] and 24, we will show that Algorithm 1 converges to a stationary point. For convenience, we set

$$
F(X):=\lambda\left(\operatorname{Tr}(X)-\|X\|_{F}\right)+\mu\|X\|_{1}+\frac{1}{2}\|\mathcal{A}(X)-\mathbf{b}\|_{2}^{2}
$$

We first show that $\left\{F\left(X^{k}\right)\right\}_{k \geq 1}$ generated by Algorithm 1 is a monotonically decreasing sequence.

Lemma 5.1. If $\left\{X^{k}\right\}_{k \geq 1}$ is a sequence generated by Algorithm 1, then we have

$$
F\left(X^{k}\right)-F\left(X^{k+1}\right) \geq 0, \quad \text { for all } k \geq 0 .
$$


Proof. We consider the $k$ th iteration of Algorithm 1. Recall that $X^{k+1}$ is the solution to (5.1) in Algorithm 1. Set $X^{k+1}:=X_{1}^{k+1}+i X_{2}^{k+1}$ and $Y^{k}:=Y_{1}^{k}+i Y_{2}^{k}$ where $X_{1}^{k+1}, X_{2}^{k+1}, Y_{1}^{k}, Y_{2}^{k} \in \mathbb{R}^{d \times d}$. Take

$$
\begin{aligned}
& f_{1}\left(X_{1}, X_{2}\right):=\mu\left\|\left[\begin{array}{l}
\operatorname{vec}\left(X_{1}\right) \\
\operatorname{vec}\left(X_{2}\right)
\end{array}\right]\right\|_{1,2}, \\
& f_{2}\left(X_{1}, X_{2}\right):=\lambda\left(\operatorname{Tr}\left(X_{1}\right)-\left\langle\left[\begin{array}{c}
Y_{1}^{k} \\
Y_{2}^{k}
\end{array}\right],\left[\begin{array}{l}
X_{1} \\
X_{2}
\end{array}\right]\right\rangle\right)+\frac{1}{2}\left\|\widetilde{\mathcal{A}}\left(\left[\begin{array}{l}
X_{1} \\
X_{2}
\end{array}\right]\right)-\mathbf{b}\right\|_{2}^{2},
\end{aligned}
$$

and $\Omega:=\mathbb{H}_{+}^{d \times d}$. Then $f_{1}$ is convex, $f_{2}$ is smooth, and $T_{\Omega}\left(\left[\begin{array}{c}X_{1}^{k+1} \\ X_{2}^{k+1}\end{array}\right]\right)$ is convex. According to Proposition 4.2, we have

$$
\lambda\left(\left[\begin{array}{l}
\mathbf{I} \\
\mathbf{0}
\end{array}\right]-\left[\begin{array}{l}
Y_{1}^{k} \\
Y_{2}^{k}
\end{array}\right]\right)+\mu\left[\begin{array}{l}
G_{1}^{k+1} \\
G_{2}^{k+1}
\end{array}\right]+\widetilde{\mathcal{A}}^{*}\left(\widetilde{\mathcal{A}}\left(\left[\begin{array}{l}
X_{1}^{k+1} \\
X_{2}^{k+1}
\end{array}\right]\right)-\mathbf{b}\right)=\left[\begin{array}{l}
\Lambda_{1}^{k+1} \\
\Lambda_{2}^{k+1}
\end{array}\right],
$$

and

$$
\left\langle\left[\begin{array}{c}
\Lambda_{1}^{k+1} \\
\Lambda_{2}^{k+1}
\end{array}\right],\left[\begin{array}{l}
X_{1}^{k+1} \\
X_{2}^{k+1}
\end{array}\right]\right\rangle=0
$$

for some $\Lambda^{k+1}=\Lambda_{1}^{k+1}+i \Lambda_{2}^{k+1}$ with $\left[\begin{array}{l}\Lambda_{1}^{k+1} \\ \Lambda_{2}^{k+1}\end{array}\right] \in \mathbb{H}_{+}^{d \times d}$, and $G^{k+1}=G_{1}^{k+1}+i G_{2}^{k+1}$ with $\left[\begin{array}{l}G_{1}^{k+1} \\ G_{2}^{k+1}\end{array}\right] \in$ $\partial\left(\left\|\left[\begin{array}{c}\operatorname{vec}\left(X_{1}^{k+1}\right) \\ \operatorname{vec}\left(X_{2}^{k+1}\right)\end{array}\right]\right\|_{1,2}\right)$. According to Proposition 4.3, we have

$$
\begin{cases}{\left[G_{1}^{k+1}\right]_{i_{1}, i_{2}}^{2}+\left[G_{2}^{k+1}\right]_{i_{1}, i_{2}}^{2} \leq 1,} & \text { if }\left[X_{1}^{k+1}\right]_{i_{1}, i_{2}}=\left[X_{2}^{k+1}\right]_{i_{1}, i_{2}}=0 \\ \left(\left[G_{1}^{k+1}\right]_{i_{1}, i_{2}},\left[G_{2}^{k+1}\right]_{i_{1}, i_{2}}\right)=\frac{\left(\left[X_{1}^{k+1}\right]_{i_{1}, i_{2}},\left[X_{2}^{k+1}\right]_{i_{1}, i_{2}}\right)}{\sqrt{\left[X_{1}^{k+1}\right]_{i_{1}, i_{2}}^{2}+\left[X_{2}^{k+1}\right]_{i_{1}, i_{2}}^{2}}}, & \text { otherwise. }\end{cases}
$$

Using a similar method for proving Lemma 4.4, we can obtain (5.6). According to (5.5), we have

$$
\left\langle\left[\begin{array}{l}
X_{1}^{k}-X_{1}^{k+1} \\
X_{2}^{k}-X_{2}^{k+1}
\end{array}\right], \lambda\left(\left[\begin{array}{l}
\mathbf{I} \\
\mathbf{0}
\end{array}\right]-\left[\begin{array}{l}
Y_{1}^{k} \\
Y_{2}^{k}
\end{array}\right]\right)+\mu\left[\begin{array}{l}
G_{1}^{k+1} \\
G_{2}^{k+1}
\end{array}\right]+\widetilde{\mathcal{A}}^{*}\left(\widetilde{\mathcal{A}}\left(\left[\begin{array}{c}
X_{1}^{k+1} \\
X_{2}^{k+1}
\end{array}\right]\right)-\mathbf{b}\right)\right\rangle=\left\langle\left[\begin{array}{c}
X_{1}^{k}-X_{1}^{k+1} \\
X_{2}^{k}-X_{2}^{k+1}
\end{array}\right],\left[\begin{array}{c}
\Lambda_{1}^{k+1} \\
\Lambda_{2}^{k+1}
\end{array}\right]\right\rangle .
$$

Combining (5.7) and

$$
\left\langle\left[\begin{array}{l}
X_{1}^{k+1} \\
X_{2}^{k+1}
\end{array}\right],\left[\begin{array}{l}
G_{1}^{k+1} \\
G_{2}^{k+1}
\end{array}\right]\right\rangle=\left\|X^{k+1}\right\|_{1},\left\langle\left[\begin{array}{c}
X_{1}^{k} \\
X_{2}^{k}
\end{array}\right],\left[\begin{array}{l}
Y_{1}^{k} \\
Y_{2}^{k}
\end{array}\right]\right\rangle=\left\|X^{k}\right\|_{F},\left\langle\left[\begin{array}{c}
\Lambda_{1}^{k+1} \\
\Lambda_{2}^{k+1}
\end{array}\right],\left[\begin{array}{l}
X_{1}^{k+1} \\
X_{2}^{k+1}
\end{array}\right]\right\rangle=0,
$$

we obtain that

$$
\begin{aligned}
\left\langle X^{k}, \Lambda^{k+1}\right\rangle= & \lambda \operatorname{Tr}\left(X^{k}-X^{k+1}\right)-\lambda\left\|X^{k}\right\|_{F}+\lambda\left\langle X^{k+1}, Y^{k}\right\rangle \\
& +\mu\left\langle X^{k}, G^{k+1}\right\rangle-\mu\left\|X^{k+1}\right\|_{1}+\left\langle\mathcal{A}\left(X^{k}-X^{k+1}\right), \mathcal{A}\left(X^{k+1}\right)-\mathbf{b}\right\rangle,
\end{aligned}
$$

since $\widetilde{\mathcal{A}}\left(\left[\begin{array}{c}X_{1}^{k+1} \\ X_{2}^{k+1}\end{array}\right]\right)=\mathcal{A}\left(X^{k+1}\right)$ and $\widetilde{\mathcal{A}}\left(\left[\begin{array}{c}X_{1}^{k}-X_{1}^{k+1} \\ X_{2}^{k}-X_{2}^{k+1}\end{array}\right]\right)=\mathcal{A}\left(X^{k}-X^{k+1}\right)$ with $X^{k}=X_{1}^{k}+i X_{2}^{k}$ and $X^{k+1}=X_{1}^{k+1}+i X_{2}^{k+1}$. Combining

$$
\begin{aligned}
F\left(X^{k}\right)-F\left(X^{k+1}\right)= & \frac{1}{2}\left\|\mathcal{A}\left(X^{k+1}-X^{k}\right)\right\|_{2}^{2}+\left\langle\mathcal{A}\left(X^{k}-X^{k+1}\right), \mathcal{A}\left(X^{k+1}\right)-\mathbf{b}\right\rangle \\
& +\mu\left(\left\|X^{k}\right\|_{1}-\left\|X^{k+1}\right\|_{1}\right)+\lambda\left(\operatorname{Tr}\left(X^{k}-X^{k+1}\right)-\left\|X^{k}\right\|_{F}+\left\|X^{k+1}\right\|_{F}\right),
\end{aligned}
$$


and (5.8), we arrive at

$$
\begin{aligned}
F\left(X^{k}\right)-F\left(X^{k+1}\right)= & \frac{1}{2}\left\|\mathcal{A}\left(X^{k+1}-X^{k}\right)\right\|_{2}^{2}+\mu\left(\left\|X^{k}\right\|_{1}-\left\langle X^{k}, G^{k+1}\right\rangle\right)+\left\langle X^{k}, \Lambda^{k+1}\right\rangle \\
& +\lambda\left(\left\|X^{k+1}\right\|_{F}-\left\langle X^{k+1}, Y^{k}\right\rangle\right) \\
\geq & 0 .
\end{aligned}
$$

Here, the last inequality follows from $\left\|X^{k}\right\|_{1}-\left\langle X^{k}, G^{k+1}\right\rangle \geq 0,\left\|X^{k+1}\right\|_{F}-\left\langle X^{k+1}, Y^{k}\right\rangle \geq 0$, and $\left\langle X^{k}, \Lambda^{k+1}\right\rangle \geq 0$ since $\left\|G^{k+1}\right\|_{\infty} \leq 1,\left\|Y^{k}\right\|_{F} \leq 1$, and $\Lambda^{k+1} \succeq 0$.

We next show the convergence property of Algorithm 1

Theorem 5.2. Assume that $\left\{X^{k}\right\}_{k \geq 1}$ is a sequence generated by Algorithm 1, We have

(1) $\left\{X^{k}\right\}_{k \geq 1}$ is a bounded sequence;

(2) $\lim _{k \rightarrow \infty}\left\|X^{k+1}-X^{k}\right\|_{F}=0$;

(3) Assume that $\widetilde{X}=\widetilde{X}_{1}+i \widetilde{X}_{2}$ is an accumulation point of $\left\{X^{k}\right\}_{k \geq 1}$. Then $\tilde{X}$ satisfies:

(i) Stationary condition:

$$
\lambda\left(\left[\begin{array}{l}
\mathbf{I} \\
\mathbf{0}
\end{array}\right]-\left[\begin{array}{c}
\widetilde{X}_{1} \\
\widetilde{X}_{2}
\end{array}\right] /\left\|\left[\begin{array}{c}
\widetilde{X}_{1} \\
\widetilde{X}_{2}
\end{array}\right]\right\|_{F}\right)+\mu\left[\begin{array}{l}
\widetilde{G}_{1} \\
\widetilde{G}_{2}
\end{array}\right]+\widetilde{\mathcal{A}}^{*}\left(\widetilde{\mathcal{A}}\left(\left[\begin{array}{c}
\widetilde{X}_{1} \\
\widetilde{X}_{2}
\end{array}\right]\right)-\mathbf{b}\right)-\left[\begin{array}{l}
\widetilde{\Lambda}_{1} \\
\widetilde{\Lambda}_{2}
\end{array}\right]=0 ;
$$

(ii) Complementary slackness condition:

$$
\left\langle\left[\begin{array}{l}
\widetilde{\Lambda}_{1} \\
\widetilde{\Lambda}_{2}
\end{array}\right],\left[\begin{array}{l}
\widetilde{X}_{1} \\
\widetilde{X}_{2}
\end{array}\right]\right\rangle=0
$$

$$
\text { for some }\left[\begin{array}{l}
\widetilde{\Lambda}_{1} \\
\widetilde{\Lambda}_{2}
\end{array}\right] \in \mathbb{H}_{+}^{d \times d} \text { and }
$$

$$
\left[\begin{array}{l}
\widetilde{G}_{1} \\
\widetilde{G}_{2}
\end{array}\right] \in \partial\left(\left\|\left[\begin{array}{c}
\operatorname{vec}\left(\widetilde{X}_{1}\right) \\
\operatorname{vec}\left(\widetilde{X}_{2}\right)
\end{array}\right]\right\|_{1,2}\right)
$$

where $\partial\left(\left\|\left[\begin{array}{l}\operatorname{vec}\left(\widetilde{X}_{1}\right) \\ \operatorname{vec}\left(\widetilde{X}_{2}\right)\end{array}\right]\right\|_{1,2}\right)$ is given in 4.5).

Proof. (1) The definition of $F$ implies $\mu\left\|X^{k+1}\right\|_{1} \leq F\left(X^{k+1}\right)$ and hence $\left\|X^{k+1}\right\|_{1} \leq F\left(X^{k+1}\right) / \mu \leq$ $F\left(X^{0}\right) / \mu$ for $k \geq 1$. Here we use Lemma [5.1, i.e., $\left\{F\left(X^{k}\right)\right\}_{k \geq 1}$ is monotonically decreasing. Hence, $\left\{X^{k}\right\}_{k \geq 1}$ is a bounded sequence.

(2) We first consider the case where $X^{1}=\mathbf{0}$. A simple calculation shows that $X^{k}=\mathbf{0}$ for $k \geq 2$ provided that $X^{1}=\mathbf{0}$, and we arrive at the conclusion immediately. So, we next just consider the case on $X^{1} \neq \mathbf{0}$. Taking $k=0$ in (5.9), we obtain that

$$
F\left(X^{0}\right)-F\left(X^{1}\right)=F(\mathbf{0})-F\left(X^{1}\right)=\frac{1}{2}\left\|\mathcal{A}\left(X^{1}\right)\right\|_{2}^{2}+\lambda\left\|X^{1}\right\|_{F} \geq \lambda\left\|X^{1}\right\|_{F}>0
$$

as $Y^{k}=\mathbf{0}$. It implies $F\left(X^{k}\right) \leq F\left(X^{1}\right)<F(\mathbf{0})$ for any $k \geq 2$. Hence, we obtain that $X^{k} \neq 0$ for all $k \geq 1$. By (5.9), we obtain that

$$
F\left(X^{k}\right)-F\left(X^{k+1}\right) \geq \frac{1}{2}\left\|\mathcal{A}\left(X^{k+1}-X^{k}\right)\right\|_{2}^{2}+\lambda\left(\left\|X^{k+1}\right\|_{F}-\left\langle X^{k+1}, Y^{k}\right\rangle\right) .
$$


Noting that $\left\{F\left(X^{k}\right)\right\}_{k \geq 1}$ is a convergent sequence and $\left\|X^{k+1}\right\|_{F}-\left\langle X^{k+1}, Y^{k}\right\rangle \geq 0$, we have

$$
\lim _{k \rightarrow \infty}\left\|\mathcal{A}\left(X^{k}-X^{k+1}\right)\right\|_{2}=0
$$

and

$$
\lim _{k \rightarrow \infty}\left(\left\|X^{k+1}\right\|_{F}-\left\langle X^{k+1}, Y^{k}\right\rangle\right)=\lim _{k \rightarrow \infty}\left(\left\|X^{k+1}\right\|_{F}-\left\langle X^{k+1}, \frac{X^{k}}{\left\|X^{k}\right\|_{F}}\right\rangle\right)=0 .
$$

The following argument is similar with that in Proposition 3.1 (b) in 24. We put it here for completeness. Set $c_{k}:=\frac{\left\langle X^{k}, X^{k+1}\right\rangle}{\left\|X^{k}\right\|_{F}^{2}}$ and $E^{k}:=X^{k+1}-c_{k} X^{k}$. It suffices to prove that $E^{k} \rightarrow \mathbf{0}$ and $c_{k} \rightarrow 1$. According to (5.14) and boundness of $\left\{X^{k}\right\}_{k \geq 1}$, we have

$$
\left\|E^{k}\right\|_{F}^{2}=\left\|X^{k+1}\right\|_{F}^{2}-\frac{\left\langle X^{k}, X^{k+1}\right\rangle^{2}}{\left\|X^{k}\right\|_{F}^{2}}=\left(\left\|X^{k+1}\right\|_{F}-\frac{\left\langle X^{k}, X^{k+1}\right\rangle}{\left\|X^{k}\right\|_{F}^{2}}\right)\left(\left\|X^{k+1}\right\|_{F}+\frac{\left\langle X^{k}, X^{k+1}\right\rangle}{\left\|X^{k}\right\|_{F}^{2}}\right) \rightarrow 0,
$$

Then we have

$$
0=\lim _{k \rightarrow \infty}\left\|\mathcal{A}\left(X^{k}-X^{k+1}\right)\right\|_{2}=\lim _{k \rightarrow \infty}\left\|\mathcal{A}\left(\left(c_{k}-1\right) X^{k}-E^{k}\right)\right\|_{2}=\lim _{k \rightarrow \infty}\left|c_{k}-1\right|\left\|\mathcal{A}\left(X^{k}\right)\right\|_{2} .
$$

If $\lim _{k \rightarrow \infty} c_{k} \neq 1$, then there exists a subsequence $\left\{X^{k_{j}}\right\}$ such that $\left\|\mathcal{A}\left(X^{k_{j}}\right)\right\|_{2} \rightarrow 0$. Therefore, we can obtain that

$$
\lim _{k_{j} \rightarrow \infty} F\left(X^{k_{j}}\right) \geq \lim _{k_{j} \rightarrow \infty} \frac{1}{2}\left\|\mathcal{A}\left(X^{k_{j}}\right)-\mathbf{b}\right\|_{2}^{2}=\frac{1}{2}\|\mathbf{b}\|_{2}^{2}=F\left(X^{0}\right)
$$

which leads to a contradiction to the fact that

$$
F\left(X^{k_{j}}\right) \leq F\left(X^{1}\right)<F\left(X^{0}\right) .
$$

Thus we can get $c_{k} \rightarrow 1, E^{k} \rightarrow \mathbf{0}$, and thus $X^{k+1}-X^{k} \rightarrow \mathbf{0}$, when $k \rightarrow \infty$.

(3) Assume that $\left\{X^{k_{j}}\right\}_{j \geq 1} \subset\left\{X^{k}\right\}_{k \geq 1}$ is a subsequence satisfying $\lim _{j \rightarrow \infty} X^{k_{j}}=\widetilde{X}=\widetilde{X}_{1}+i \widetilde{X}_{2} \neq$ 0. For simplicity, we abuse the notation and denote $\left\{X^{k_{j}}\right\}$ as $\left\{X^{j}\right\}$. Replacing $k$ by $j-1$ in (5.5) and (5.6), we have

$$
\lambda\left(\left[\begin{array}{l}
\mathbf{I} \\
\mathbf{0}
\end{array}\right]-\left[\begin{array}{l}
Y_{1}^{j-1} \\
Y_{2}^{j-1}
\end{array}\right]\right)+\mu\left[\begin{array}{l}
G_{1}^{j} \\
G_{2}^{j}
\end{array}\right]+\widetilde{\mathcal{A}}^{*}\left(\widetilde{\mathcal{A}}\left(\left[\begin{array}{l}
X_{1}^{j} \\
X_{2}^{j}
\end{array}\right]\right)-\mathbf{b}\right)=\left[\begin{array}{l}
\Lambda_{1}^{j} \\
\Lambda_{2}^{j}
\end{array}\right],
$$

and

$$
\left\langle\left[\begin{array}{c}
\Lambda_{1}^{j} \\
\Lambda_{2}^{j}
\end{array}\right],\left[\begin{array}{l}
X_{1}^{j} \\
X_{2}^{j}
\end{array}\right]\right\rangle=0
$$

for some $\Lambda^{j}=\Lambda_{1}^{j}+i \Lambda_{2}^{j}$ with $\left[\begin{array}{c}\Lambda_{1}^{j} \\ \Lambda_{2}^{j}\end{array}\right] \in \mathbb{H}_{+}^{d \times d}$, and $G^{j}=G_{1}^{j}+i G_{2}^{j}$ with

$$
\left[\begin{array}{l}
G_{1}^{j} \\
G_{2}^{j}
\end{array}\right] \in \partial\left(\left\|\left[\begin{array}{l}
\operatorname{vec}\left(X_{1}^{j}\right) \\
\operatorname{vec}\left(X_{2}^{j}\right)
\end{array}\right]\right\|_{1,2}\right)
$$

Note that (5.15) is equivalent to

$$
\lambda\left(\left[\begin{array}{l}
\mathbf{I} \\
\mathbf{0}
\end{array}\right]-\left[\begin{array}{l}
Y_{1}^{j-1} \\
Y_{2}^{j-1}
\end{array}\right]\right)+\widetilde{\mathcal{A}}^{*}\left(\widetilde{\mathcal{A}}\left(\left[\begin{array}{l}
X_{1}^{j} \\
X_{2}^{j}
\end{array}\right]\right)-\mathbf{b}\right)=\left[\begin{array}{l}
\Lambda_{1}^{j} \\
\Lambda_{2}^{j}
\end{array}\right]-\mu\left[\begin{array}{l}
G_{1}^{j} \\
G_{2}^{j}
\end{array}\right] .
$$

Noting that

$$
\lim _{j \rightarrow \infty}\left[\begin{array}{l}
Y_{1}^{j-1} \\
Y_{2}^{j-1}
\end{array}\right]=\left[\begin{array}{l}
\widetilde{X}_{1} \\
\widetilde{X}_{2}
\end{array}\right] /\left\|\left[\begin{array}{c}
\widetilde{X}_{1} \\
\widetilde{X}_{2}
\end{array}\right]\right\|_{F},
$$


we obtain that the left hand side of (5.18) converges to

$$
\lim _{j \rightarrow \infty} \lambda\left(\left[\begin{array}{l}
\mathbf{I} \\
\mathbf{0}
\end{array}\right]-\left[\begin{array}{c}
Y_{1}^{j-1} \\
Y_{2}^{j-1}
\end{array}\right]\right)+\widetilde{\mathcal{A}}^{*}\left(\widetilde{\mathcal{A}}\left(\left[\begin{array}{l}
X_{1}^{j} \\
X_{2}^{j}
\end{array}\right]\right)-\mathbf{b}\right)=\lambda\left(\left[\begin{array}{l}
\mathbf{I} \\
\mathbf{0}
\end{array}\right]-\left[\begin{array}{c}
\widetilde{X}_{1} \\
\widetilde{X}_{2}
\end{array}\right] /\left\|\left[\begin{array}{l}
\widetilde{X}_{1} \\
\widetilde{X}_{2}
\end{array}\right]\right\|_{F}\right)+\widetilde{\mathcal{A}}^{*}\left(\widetilde{\mathcal{A}}\left(\left[\begin{array}{c}
\widetilde{X}_{1} \\
\widetilde{X}_{2}
\end{array}\right]\right)-\mathbf{b}\right) .
$$

For convenience, we set

$$
P^{j}:=\left[\begin{array}{l}
\Lambda_{1}^{j} \\
\Lambda_{2}^{j}
\end{array}\right] \quad \text { and } \quad Q^{j}:=-\mu\left[\begin{array}{c}
G_{1}^{j} \\
G_{2}^{j}
\end{array}\right] .
$$

According to (5.17) and Proposition [4.3, we have $\left\|G_{1}^{j}\right\|_{\infty} \leq 1$ and $\left\|G_{2}^{j}\right\|_{\infty} \leq 1$. Combining (5.18) and the boundedness of $\left\{X^{j}\right\}_{j \geq 1}$, we obtain that $\left\{P^{j}\right\}_{j \geq 1}$ and $\left\{Q^{j}\right\}_{j \geq 1}$ are also bounded sequences, which can belong to some compact sets $S \subset \mathbb{H}_{+}^{d \times d}$ and $T$, respectively.

We assume that $\left\{j_{l}\right\}_{l \geq 1}$ is a subsequence of $\{j\}_{j \geq 1}$ such that $\lim _{l \rightarrow \infty} P_{j_{l}}=\widetilde{P}$ and $\lim _{l \rightarrow \infty} Q_{j_{l}}=\widetilde{Q}$ for some $\widetilde{P} \in S, \widetilde{Q} \in T$. More concretely, we have

$$
\lim _{l \rightarrow \infty} P^{j_{l}}=\lim _{l \rightarrow \infty}\left[\begin{array}{l}
\Lambda_{1}^{j_{l}} \\
\Lambda_{2}^{j_{l}}
\end{array}\right]=\left[\begin{array}{l}
\widetilde{\Lambda}_{1} \\
\widetilde{\Lambda}_{2}
\end{array}\right] \text {, and } \lim _{l \rightarrow \infty} Q^{j_{l}}=\lim _{l \rightarrow \infty}-\mu\left[\begin{array}{c}
G_{1}^{j_{l}} \\
G_{2}^{j_{l}}
\end{array}\right]=-\mu\left[\begin{array}{c}
\widetilde{G}_{1} \\
\widetilde{G}_{2}
\end{array}\right]
$$

for some

$$
\left[\begin{array}{l}
\widetilde{\Lambda}_{1} \\
\widetilde{\Lambda}_{2}
\end{array}\right] \in S \subset \mathbb{H}_{+}^{d \times d}
$$

According to (5.18), we have

$$
\lambda\left(\left[\begin{array}{l}
\mathbf{I} \\
\mathbf{0}
\end{array}\right]-\left[\begin{array}{c}
\widetilde{X}_{1} \\
\widetilde{X}_{2}
\end{array}\right] /\left\|\left[\begin{array}{l}
\widetilde{X}_{1} \\
\widetilde{X}_{2}
\end{array}\right]\right\|_{F}\right)+\widetilde{\mathcal{A}}^{*}\left(\widetilde{\mathcal{A}}\left(\left[\begin{array}{c}
\widetilde{X}_{1} \\
\widetilde{X}_{2}
\end{array}\right]\right)-\mathbf{b}\right)=\left[\begin{array}{c}
\widetilde{\Lambda}_{1} \\
\widetilde{\Lambda}_{2}
\end{array}\right]-\mu\left[\begin{array}{l}
\widetilde{G}_{1} \\
\widetilde{G}_{2}
\end{array}\right],
$$

which implies the stationary condition (5.10). The complementary slackness condition (5.11) is obtained by

$$
\left\langle\left[\begin{array}{c}
\widetilde{\Lambda}_{1} \\
\widetilde{\Lambda}_{2}
\end{array}\right],\left[\begin{array}{c}
\widetilde{X}_{1} \\
\widetilde{X}_{2}
\end{array}\right]\right\rangle=\lim _{l \rightarrow \infty}\left\langle\left[\begin{array}{c}
\Lambda_{1}^{j_{l}} \\
\Lambda_{2}^{j_{l}}
\end{array}\right],\left[\begin{array}{c}
X_{1}^{j_{l}} \\
X_{2}^{j_{l}}
\end{array}\right]\right\rangle=0 .
$$

Here, we use (5.16).

We remain to prove (5.12). For sufficiently large $j_{l}$, we have $\operatorname{supp}(\tilde{X}) \subset \operatorname{supp}\left(X^{j_{l}}\right)$. If $\left(i_{1}, i_{2}\right) \in$ $\operatorname{supp}(\widetilde{X})$, then

$$
\lim _{l \rightarrow \infty}\left(\left[G_{1}^{j_{l}}\right]_{i_{1}, i_{2}},\left[G_{2}^{j_{l}}\right]_{i_{1}, i_{2}}\right)=\frac{\left(\left[\widetilde{X}_{1}\right]_{i_{1}, i_{2}},\left[\widetilde{X}_{2}\right]_{i_{1}, i_{2}}\right)}{\sqrt{\left[\widetilde{X}_{1}\right]_{i_{1}, i_{2}}^{2}+\left[\widetilde{X}_{2}\right]_{i_{1}, i_{2}}^{2}}} .
$$

If $\left(i_{1}, i_{2}\right) \notin \operatorname{supp}(\widetilde{X})$, we have

$$
\left(\left[G_{1}^{j_{l}}\right]_{i_{1}, i_{2}}\right)^{2}+\left(\left[G_{2}^{j_{l}}\right]_{i_{1}, i_{2}}\right)^{2} \leq 1
$$

and hence

$$
\left(\left[\widetilde{G}_{1}\right]_{i_{1}, i_{2}}\right)^{2}+\left(\left[\widetilde{G}_{2}\right]_{i_{1}, i_{2}}\right)^{2} \leq 1 .
$$

Thus

$$
\lim _{l \rightarrow \infty}\left[\begin{array}{l}
G_{1}^{j_{l}} \\
G_{2}^{j_{l}}
\end{array}\right]=\left[\begin{array}{l}
\widetilde{G}_{1} \\
\widetilde{G}_{2}
\end{array}\right] \in \partial\left(\left\|\left[\begin{array}{c}
\operatorname{vec}\left(\widetilde{X}_{1}\right) \\
\operatorname{vec}\left(\widetilde{X}_{2}\right)
\end{array}\right]\right\|_{1,2}\right)
$$

which leads to (5.12). 


\section{NumERICAL EXPERIMENTS}

The purpose of numerical experiments is to compare the performance of (1.8) with that of SPARTA [18, of SWF [25] and of SPRSF [11. We choose the parameters of those algorithms as in [18, 25, 11]. In this section, we use the relative error

$$
\text { relative error }:=\frac{d_{r}\left(\mathbf{z}, \mathbf{x}_{0}\right)}{\left\|\mathbf{x}_{0}\right\|_{2}},
$$

where $d_{r}\left(\mathbf{z}, \mathbf{x}_{0}\right)=\min \left\|\mathbf{z} \pm \mathbf{x}_{0}\right\|_{2}$ for the real case and $d_{r}(\mathbf{z}, \mathbf{x})=\min _{\theta \in[0,2 \pi)}\|\exp (-i \theta) \mathbf{z}-\mathbf{x}\|_{2}$ for the complex case. In our numerical experiments, we assume that the sampling vectors $\mathbf{a}_{j}, j=1, \ldots, m$ are Gaussian random vector, i.e., $\mathbf{a}_{j} \sim \mathcal{N}\left(0, \mathbf{I}_{d}\right)$ for real case and $\mathbf{a}_{j} \sim \mathcal{N}\left(0, \frac{1}{2} \mathbf{I}_{d}\right)+i \mathcal{N}\left(0, \frac{1}{2} \mathbf{I}_{d}\right)$ for complex case.

For each fixed $k$, the support of a $k$-sparse signal $\mathbf{x}_{0}$ is drawn from the uniform distribution over the set of all subsets of $[1, m] \cap \mathbb{Z}$ of size $k$. The non-zero entries of the real (resp. complex) $k$-sparse signal $\mathbf{x}_{0}$ have Gaussian distribution $\mathcal{N}(0,1)($ resp. $\mathcal{N}(0,1)+i \mathcal{N}(0,1))$. In order to reduce dimension effect, we normalize $\mathbf{x}_{0}$ into $\left\|\mathbf{x}_{0}\right\|_{2}=1$. All experiments are carried out on Matlab 2017 with a 3.7 $\mathrm{GHz}$ Intel Core i7-8700K and 64 GB memory.

Example 6.1. The aim of this numerical experiment is to test the success rate of Algorithm 1 against the measurement number $m$. In this example, we take $k=5$ and $d=50$. The ratio between $m$ and $d$ is varied from 0.1 to 4 , with stepsize 0.1 . We choose $\mu=0.001$ and $\lambda=\frac{\mu k}{\sqrt{2}-1}$ in Algorithm 1. We classify a recovery as a success if the relative error is less than $10^{-3}$. For each fixed $m$, we repeat the experiments for 40 trails and and calculate the success rate.

Figure 1 depicts the empirical probability of successful recovery against the measurement number $m$. The numerical results show that Algorithm 1 outperform other algorithms.

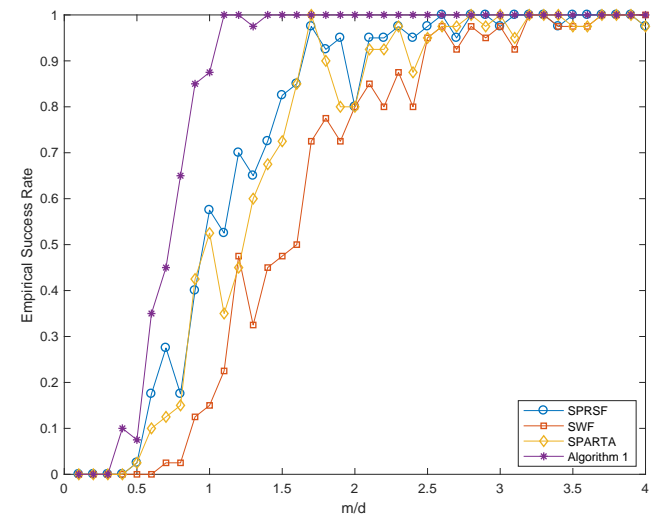

(A)

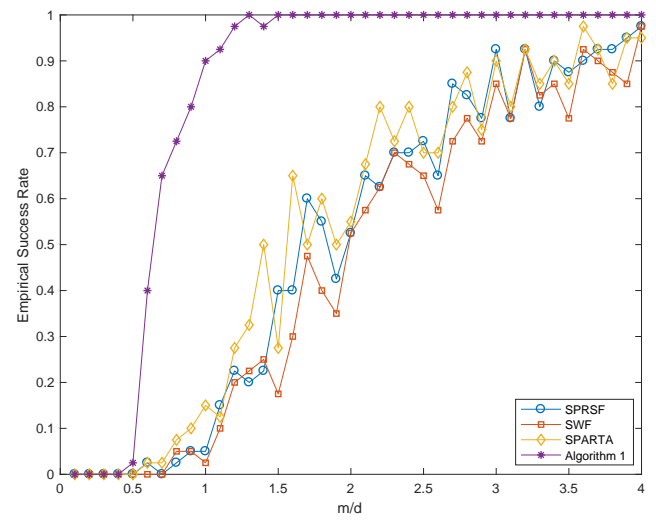

(B)

Figure 1. Comparison of different algorithms for fixed $\mathrm{k}=5$ with different $m / n$ ratio: (A) Noiseless real-valued Gaussian model; (B) Noiseless complex-valued Gaussian model. 
Example 6.2. In this example, we test the success rate of Algorithm 1 against the sparsity level $k$. We take $d=50$ and $m=2 d$. The parameters in Algorithm 1 are taken as $\mu=0.001$ and $\lambda=\frac{\mu k}{\sqrt{2}-1}$. Figure 2 depicts the numerical results. It shows that Algorithm 1 is superior to the SPRSF, SWF and SPARTA for both real and complex cases. Furthermore, we can see that Algorithm 1 can make good performance even under large level of sparsity.

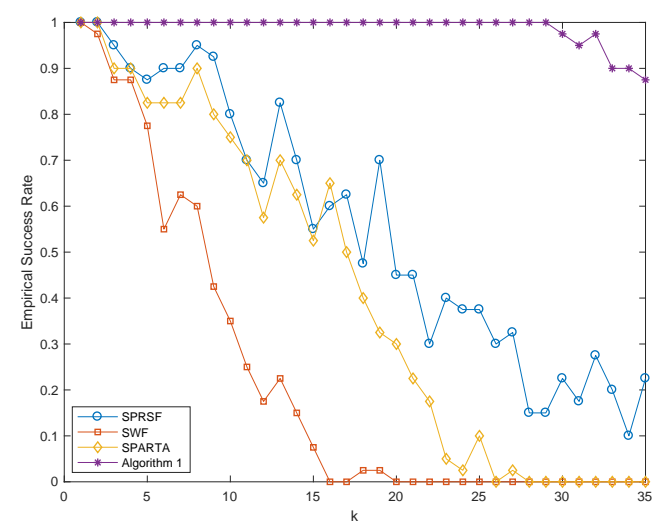

(A)

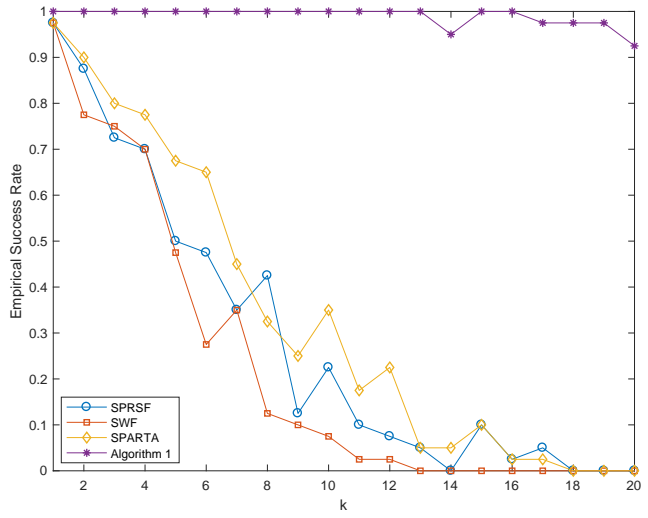

(B)

Figure 2. Comparison of different algorithms for different sparsity level $k$ : (A) Noiseless real-valued Gaussian model; (B) Noiseless complex-valued Gaussian model.

Example 6.3. In this example, we test the robustness of Algorithm 1 . We take $d=50, m=2 d$ and $k=5$ for both real and complex cases, followed by adding white Gaussian noise by MATLAB function awgn( $\mathcal{A}\left(\mathbf{x}_{0}\right)$, snr $)$, i.e., $b_{j}=\left|\left\langle\mathbf{a}_{j}, \mathbf{x}_{0}\right\rangle\right|^{2}+w_{j}, j=1, \ldots, m$ with $\mathbf{w} \sim \sqrt{\frac{\left\|\mathcal{A}\left(\mathbf{x}_{0}\right)\right\|_{2}^{2} / m}{10^{s n r / 10}}} \mathcal{N}\left(0, \mathbf{I}_{m}\right)$. Since other algorithms do not make $100 \%$ recovery under this setting, we only show the robustness performance on Algorithm 1. The SNR value varies from $10 \mathrm{~dB}$ to $50 \mathrm{~dB}$, with step-size 5dB. The SNR in each noise level is averaged over 20 independent trials. According to Theorem 1.2, we choose $\mu=\max \left\{0.5\|\mathbf{w}\|_{2}, 0.001\right\}$ and $\lambda=\frac{\mu k}{\sqrt{2}-1}$. We compute the signal-to noise ratio of reconstruction in $d B$ as $-20 \log _{10}$ (relative error). In Figure [3, it shows that Algorithm 1 yields robust recovery with respect to different noise level. In addition, the recovery error is a bitter larger for complex case.

\section{REFERENCES}

[1] Dimitri P. Bertsekas. Nonlinear programming, Athena Scientific, 1999.

[2] Dimitri P. Bertsekas, Angelia Nedic, and Asuman E. Ozdaglar. Convex Analysis and Optimization, Athena Scientific, 2003.

[3] Stephen Boyd, Neal Parikh, Eric Chu, Borja Peleato, and Jonathan Eckstein. Distributed optimization and statistical learning via the alternating direction method of multipliers. Foundations and Trends in Machine Learning, 3(1): 1-122, 2010.

[4] T. Tony Cai, Xiaodong Li, and Zongming Ma. Optimal rates of convergence for noisy sparse phase retrieval via thresholded Wirtinger flow. The Annals of Statistics, 44(5):2221-2251, 2016.

[5] E. J. Candès and X. Li, "Solving quadratic equations via PhaseLift when there are about as many equations as unknowns," Found. Comut. Math., 14(5):1017-1026, 2014. 


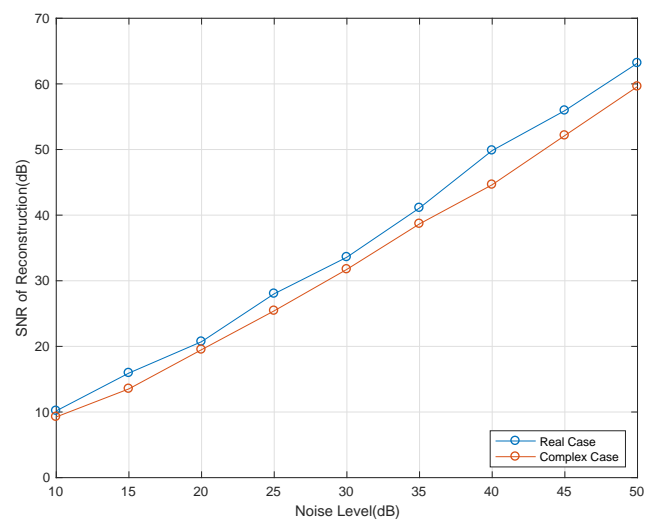

FiguRE 3. SNR of signal recovery v.s. noise level in measurements when $k=5$ : $x$-axis is the noise level varying from $10 \mathrm{db}$ to $50 \mathrm{db}, y$-axis is the reconstruction error in $\mathrm{db}$ as $-20 \log _{10}$ (relative error).

[6] Emmanuel J. Candès, Thomas Strohmer, and Vladislav Voroninski, Phaselift: Exact and stable signal recovery from magnitude measurements via convex programming. Communnications on Pure and Applied Mathematics, 66(8):1241-1274, 2013.

[7] Emmanuel J. Candès, Yonina C. Eldar, Thomas Strohmer and Vladislav Voroninski. Phase retrieval via matrix completion. SIAM Review, 57(2):225-251, 2013.

[8] Aldo Conca, Dan Edidin, Milena Hering and Cynthia Vinzant. An algebraic characterization of injectivity in phase retrieval. Applied and Computational Harmonic Analysis, 38(2):346-356, 2015.

[9] Bingsheng He and Xiaoming Yuan. On the $\mathrm{O}(1 / \mathrm{n})$ convergence rate of the Douglas-Rachford alternating direction method. SIAM Journal on Numerical Analysis, 50 (2) :700-709, 2012.

[10] Xiaodong Li and Vladislav Voroninski. Sparse Signal Recovery from Quadratic Measurements via Convex Programming, SIAM Journal on Mathematical Analysis, 45(5):3019-3033, 2013.

[11] Samuel Pinilla, Jorge Bacca and Henry Arguello. SPRSF: Sparse Phase Retrieval via Smoothing Function, arXiv: 1807.09703

[12] Yoav Shechtman, Yonina C. Eldar, Alexander Szameit and Mordechai Segev. Sparsity based sub-wavelength imaging with partially incoherent light via quadratic compressed sensing. Optics express, 19(16):14807-14822, 2011.

[13] Yoav Shechtman, Amir Beck and Yonina C. Eldar. GESPAR: Efficient phase retrieval of sparse signals. IEEE transactions on signal processing, 62(4): 928-938, 2014.

[14] P. D. Tao and L. T. H. An, Convex analysis approach to dc programming: Theory, algorithms and applications. Acta Math. Vietnam, 22: 289-355, 1997.

[15] Pham D. Tao and Le T. H. An. A D.C. optimization algorithm for solving the trust-region subproblem, SIAM Journal on Optimization, 8(2):476-505, 1988.

[16] R. Vershynin. Introduction to the non-asymptotic analysis of random matrices. In Y. C. Eldar and G. Kutyniok, editors, Compressed Sensing: Theory and Applications. Cambridge University Press, 2010. Preprint available at http://www-personal.umich.edu/ romanv/papers/papers.html

[17] Vladislav Voroninski and Zhiqiang Xu. A strong restricted isometry property, with an application to phaseless compressed sensing. Applied Computational Harmonic Analysis, 40(2):386-395, 2016.

[18] Gang Wang, Liang Zhang and Georgios B. Giannakis. Sparse Phase Retrieval via Truncated Amplitude Flow. IEEE Transactions on Signal Processing, 66(2):479-491, 2016.

[19] Yang Wang and Zhiqiang Xu. Phase retrieval for sparse signals. Applied and Computational Harmonic Analysis, 37(3): 531-544, 2014.

[20] Yang Wang and Zhiqiang Xu. Generalized phase retrieval: measurement number, matrix recovery and beyond. Applied and Computational Harmonic Analysis, 47(2):423-446, 2019.

[21] Yu Xia and Song Li. Identifiability of Multichannel Blind Deconvolution and Nonconvex Regularization Algorithm. IEEE Transactions on Signal Processing, 66(20): 5299-5312, 2018. 
[22] Yu Xia and Zhiqiang Xu. The recovery of complex sparse signals from few phaseless measurements. To appear in Applied and Computational Harmonic Analysis.

[23] Penghang Yin and Jack Xin. PhaseLiftOff: an Accurate and Stable Phase Retrieval Method Based on Difference of Trace and Frobenius Norms. Communications in Mathematical Sciences, DOI: 10.4310/CMS.2015.v13.n4.a10, 2015.

[24] Penghang Yin, Yifei Lou, Qi He and Jack Xin. Minimization of $\ell_{1-2}$ for compressed sensing. SIAM Journal on Scientific Computing, 37(1):A536-A563, 2015.

[25] Ziyang Yuan, Hongxia Wang and Qi Wang. Phase Retrieval via Sparse Wirtinger Flow. Journal of Computational and Applied Mathematics, 355: 162-173, 2019.

Department of Mathematics, Hangzhou Normal University, Hangzhou 311121, China

E-mail address: yxia@hznu.edu.cn

LSEC, Inst. Comp. Math., Academy of Mathematics and System Science, Chinese Academy of Sciences, BEIJING, 100091, ChINA

School of Mathematical Sciences, University of Chinese Academy of Sciences, Beijing 100049, China

E-mail address: xuzq@isec.cc.ac.cn 